Article

\title{
Introduction of Aureobasidium pullulans to the Phyllosphere of Organically Grown Strawberries with Focus on Its Establishment and Interactions with the Resident Microbiome
}

\author{
Justine Sylla ${ }^{1,2,3 *}$, Beatrix W. Alsanius ${ }^{3}$, Erika Krüger ${ }^{2}$, Annette Reineke ${ }^{1}$, \\ Monika Bischoff-Schaefer ${ }^{1}$ and Walter Wohanka ${ }^{1}$
}

1 Department of Phytomedicine, Geisenheim University, Von-Lade-Str. 1, Geisenheim DE-65366, Germany; E-Mails: Annette.Reineke@hs-gm.de (A.R.);

Monika.Bischoff-Schaefer@hs-gm.de (M.B.-S.); Walter.Wohanka@hs-gm.de (W.W.)

2 Department of Pomology, Geisenheim University, Von-Lade-Str. 1, Geisenheim DE-65366, Germany; E-Mail: Erika.Krueger@hs-gm.de

3 Department of Biosystems and Technology, Swedish University of Agricultural Sciences, P.O. Box 103, Alnarp SE-23053, Sweden; E-Mail: Beatrix.Alsanius@slu.se

* Author to whom correspondence should be addressed; E-Mail: Justine.Sylla@hs-gm.de; Tel.: +49-6722-502-422; Fax: +49-6722-502-410.

Received: 31 July 2013; in revised form: 2 October 2013 / Accepted: 14 October 2013 / Published: 30 October 2013

\begin{abstract}
Currently, there is little knowledge of the establishment of repeatedly applied biological control agents (BCAs) in the phyllosphere of plants and, in particular, their interactions with the resident microbiome. Under field conditions, the BCA Aureobasidium pullulans was applied as a model organism to organically grown strawberries during two subsequent years $(2011,2012)$, either as single strain treatment or with the co-application of the entomopathogenic fungus Beauveria bassiana. Fungal and bacterial communities of strawberry leaves were investigated by means of plate counts and 454 pyrosequencing. The establishment of the introduced $A$. pullulans strains considerably differed between the two years, presumably due to distinct environmental conditions. Short-term and long-term effects of BCA applications on the composition and diversity of fungal communities could be observed as a result of successful establishment of $A$. pullulans, in 2011, showing, for instance, reduced diversity of fungal communities by competitive displacement shortly after BCA introduction. Due to considerable dynamics in untreated resident microbial
\end{abstract}


communities in the phyllosphere in general, however, we suggest that even the effects caused by the applied BCA preparations in 2011 are negligible under practical conditions.

Keywords: biological control agents: BCAs; microbial communities; organic farming; plate counts; 454 pyrosequencing

\section{Introduction}

Despite a vast amount of research on phyllosphere applied biological control agents (BCAs) (e.g., [1-6]), control of foliar pathogens is still challenging. The introduced BCAs meet a harsh environment due to fluctuating abiotic conditions (temperature, precipitation, wind and thereby drought, water availability, light spectrum) [7,8] and plant related biotic factors (e.g., nutrient paucity) [7-10]. Moreover, as immigrating organisms BCAs are exposed to numerous interactions with the resident leaf microbiome (e.g., competition for space and nutrients, adhesion to existing microbial aggregates) [10-12]. As a consequence, the numbers of applied BCAs usually rapidly decline after introduction to the phyllosphere $[4,13,14]$. Therefore, BCA applications have to be repeated regularly to maintain and to build up an active population of the introduced strains in already conquered as well as in new niches, as the crop grows, capable to counteract the target foliar pathogens.

The use of BCAs is particularly interesting in crops with high economic importance such as strawberries to substitute the extensive use of chemical pesticides. In strawberries, various BCAs (e.g., Aureobasidium pullulans and Trichoderma harzianum) have shown good potential to control plant pathogens, such as Botrytis cinerea $[3,4,15,16]$. While phyllosphere applied BCAs must employ tolerance strategies to cope with the conditions prevailing at the site of introduction [10], they should not harm the non-target microbiota, e.g., through competitive displacement [17]. They, therefore, need to be considered as transient organisms.

However, so far only a few research papers consider the fact that the plant is a dynamic environment from a plant physiological point of view. Leaf maturation and ageing have a direct impact on the physical niches, but also on nutrient availability and, consequently, alter the microbial community structure and its function [18-20]. Until now, only the temporary effects of a few BCAs on the indigenous microbial communities were detected in the environment of the phyllosphere [21,22].

We, therefore, investigated the shifts of introduced BCAs and of the culturable and non-culturable microbiota on strawberry leaves at different phenological stages, under field conditions, using culture-dependent (viable count) and culture-independent (454 pyrosequencing) methods. The yeast-like fungus $A$. pullulans served as a model BCA and was introduced as a single strain treatment or co-inoculated with the entomopathogenic fungus Beauveria bassiana. The objectives were to (i) characterize the non-manipulated microbiome of strawberry leaves in relation to the crop's growth and development, and to (ii) study the shifts in the resident phyllosphere microbiome in relation to the introduction of the model BCA in terms of short- or long-term impacts. 


\section{Results and Discussion}

Cook et al. [17] postulated that biological control agents should not competitively displace the resident microbiome associated with the BCAs' site of introduction. However, only very few investigations addressed shifts of the resident phyllosphere microbiome upon introduction of BCAs [21-25]. Almost no information is available that takes the physiological progression of the crop into account. In this study, progression of strawberry as a crop was considered by sampling strawberry leaves at three distinct phenological stages, namely (i) when the first flowers were open (BBCH 60); (ii) when seeds were visible on receptacle tissue/fruit-setting (BBCH 73); and (iii) when old leaves were dying (BBCH 93). These three sampling events corroborated with temporal aspects of BCA introduction, where the first sampling considered leaves before phyllosphere application of the BCAs, the second sampling leaves after exposure to four BCA applications (short-term effects), and the third sampling leaves after four weeks of adjourned treatments (long-term effects). To the best of our knowledge, this is the first study employing both a phenological perspective to phyllosphere applied BCAs and monitoring the microbiome by a combination of plate counts and high-throughput sequencing.

By means of 454 pyrosequencing, a total of 75,140 fungal and 35,120 bacterial reads (excluding chloroplast reads) were recovered from the ITS rRNA and 16S rRNA sample pool, respectively. These reads could be clustered into 2972 fungal OTUs and 5698 bacterial OTUs. A summary on the amount of sequence reads per treatment and sampling date is displayed in Table 1 . The average number of $16 \mathrm{~S}$ rRNA sequences varied between 103 and 636 in 2011 and, thus, was comparably low as compared to ITS rRNA sequences in 2011 and 2012. This leads to the question if all bacteria of the phyllosphere microbiota were accurately reflected. For the phenological stage $\mathrm{BBCH} 73$, in 2011, some BCA-treated leaf samples did not show enough 16S reads for data analysis. Accordingly, obtained 454 pyrosequencing data were insufficient to compare microbial communities among the different treatments at this stage and are, thus, not shown. In this context, general methodological issues have to be considered as well, e.g. the efficiency of microbial release from leaves or the potential impact of sonication on culturable microorganisms.

\subsection{Composition of Resident Microbial Communities in the Strawberry Phyllosphere}

The resident phyllosphere microbiome as determined by culture-dependent and -independent techniques varied considerably between the two years of experiment, but also between the three phenological stages.

According to plate counts, the resident culturable leaf microbiota consisted of $7.76 \times 10^{4}$ to $2.09 \times$ $10^{5} \mathrm{CFU} \mathrm{g} \mathrm{DW}^{-1}$ bacteria and $1.15 \times 10^{3}$ to $1.29 \times 10^{5} \mathrm{CFU} \mathrm{g} \mathrm{DW}^{-1}$ fungi in total, as well as $3.24 \times$ $10^{3}$ to $3.24 \times 10^{4} \mathrm{CFU} \mathrm{g} \mathrm{DW}{ }^{-1}$ endospore-forming bacteria in flowering strawberry plants (BBCH 60) in 2011 and 2012 (Figure 1). At this phenological growth stage, the numbers of resident total bacteria, total fungi and endospore-forming bacteria significantly differed between 2011 and 2012, respectively. Neither Aureobasidium spp., nor Beauveria spp., were detected at this stage in 2011, but Aureobasidium spp. were found at the same stage in 2012, albeit at a low level (Figure 1). 
Table 1. Mean numbers of 454 pyrosequencing reads in differentially treated leaf samples obtained at different phenological stages in 2011 and 2012.

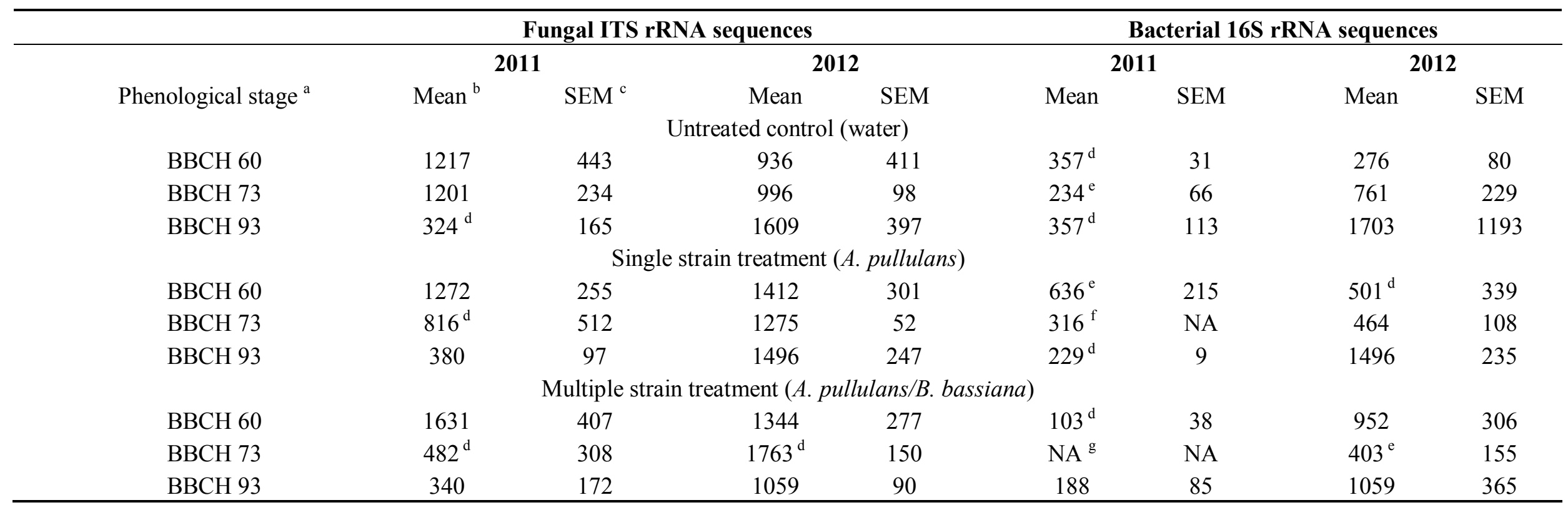

${ }^{\mathrm{a}} \mathrm{BBCH} 60$ = first flowers open; $\mathrm{BBCH} 73$ = seeds visible on receptacle tissue (fruit-setting), after four $\mathrm{BCA}$ applications in the single and multiple strain treatments; BBCH 93 = old leaves dying, four weeks after the last BCA application in the single and multiple strain treatments; ${ }^{b}$ Mean number of 454 reads per treatment (4 replicates, with 30 individual leaves per replicate); ${ }^{\mathrm{c}}$ Standard error of the mean; ${ }^{\mathrm{d}}$ Only three replicates (with $>50$ reads); ${ }^{\mathrm{e}}$ Only two replicates (with $>50$ reads);

${ }^{\mathrm{f}}$ No mean value; this treatment is only represented by one sample replicate (with $>50$ reads); ${ }^{\mathrm{g}}$ No data available (no sample replicate with $>50$ reads). 
Figure 1. Resident phyllosphere microbiome of flowering strawberries (BBCH 60) in 2011 and 2012 as determined by plate counts (pooled data, each $N=36$ ). Error bars represent SEM values. Columns marked with the same letters are not significantly different within each microbial group $(\alpha=0.05)$.

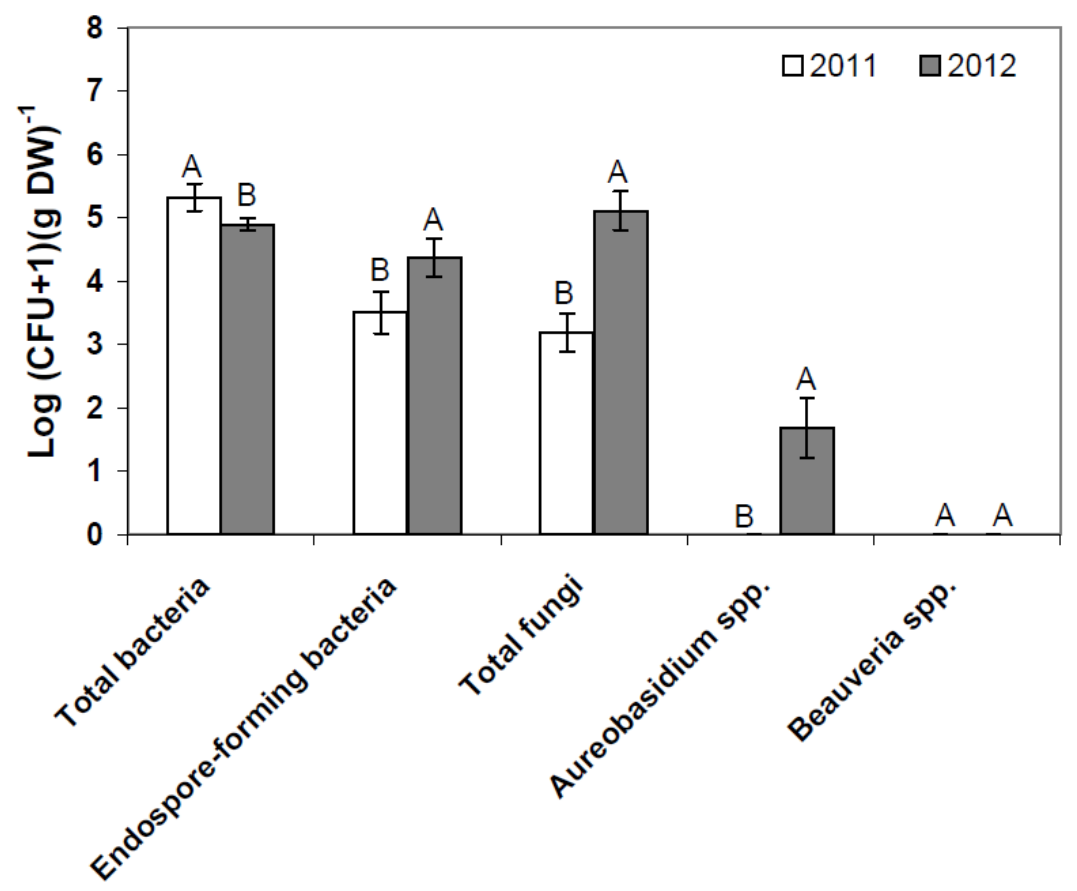

In untreated leaf samples, the amount of total resident bacteria significantly decreased $(p=0.008)$ when comparing flowering and fruit-setting strawberry plants and subsequently increased $(p=0.006)$ when old leaves died off (BBCH 93) in 2011 (see also Figure 2). In 2012, counts of total bacteria were similar in the control samples during flowering and fruit-setting and significantly increased at $\mathrm{BBCH}$ 93 ( $p=0.002)$. An increase in the abundance of culturable bacteria in decaying leaves is expected [18]. The counts of resident endospore-forming bacteria did not differ at the first two stages in 2011, but significantly decreased from the second to the third phenological stage $(p<0.001)$. In 2012, counts of endospore-forming bacteria gradually decreased from flowering plants to plants with old, dying leaves $(p<0.001)$. The amount of total resident fungi was similar at the first two phenological stages in 2011, but increased as expected on decaying leaves in $2011(p<0.001)$, which is in line with the function of fungi during decomposition [26]. In 2012, counts of total fungi were initially high during flowering and significantly decreased from flowering to the fruit-setting stage $(p<0.001)$. The amount of total fungi, however, significantly increased on decaying leaves as well ( $p<0.001)$. Interestingly, Aureobasidium spp. counts increased on leaves from untreated plots from the first through the third stage in $2011(p>0.05)$. A . pullulans is ubitiquous in the environment [27,28]. As no labeled strain was used in the treated plots, it is difficult to tell, if occurrence of $A$. pullulans on untreated strawberry leaves is due to natural inoculation or transmission by wind from the treated plots. However, Aureobasidium were detected as resident organisms on leaves on flowering plants in 2012, but not at later stages. 
Figure 2. Culturable phyllosphere microbiota in strawberries at two distinct phenological stages (BBCH 73 and $\mathrm{BBCH} 93)$ during two subsequent years $(2011,2012)$ as determined by plate counts $(N=12)$. Aureobasidium pullulans was introduced as a model organism either as a single strain treatment (AP) or co-inoculated with Beauveria bassiana (AP/BB) through commercial formulations. The two phenological stages represent short- $\mathrm{BBCH}$ 73, after four BCA applications) and long-term effects $(\mathrm{BBCH} 93$, four weeks after adjournment of BCA applications). The control (W) was treated with water from a lotic reservoir. Error bars represent SEM values. Columns marked with the same letters are not significantly different within each microbial group $(\alpha=0.05)$.
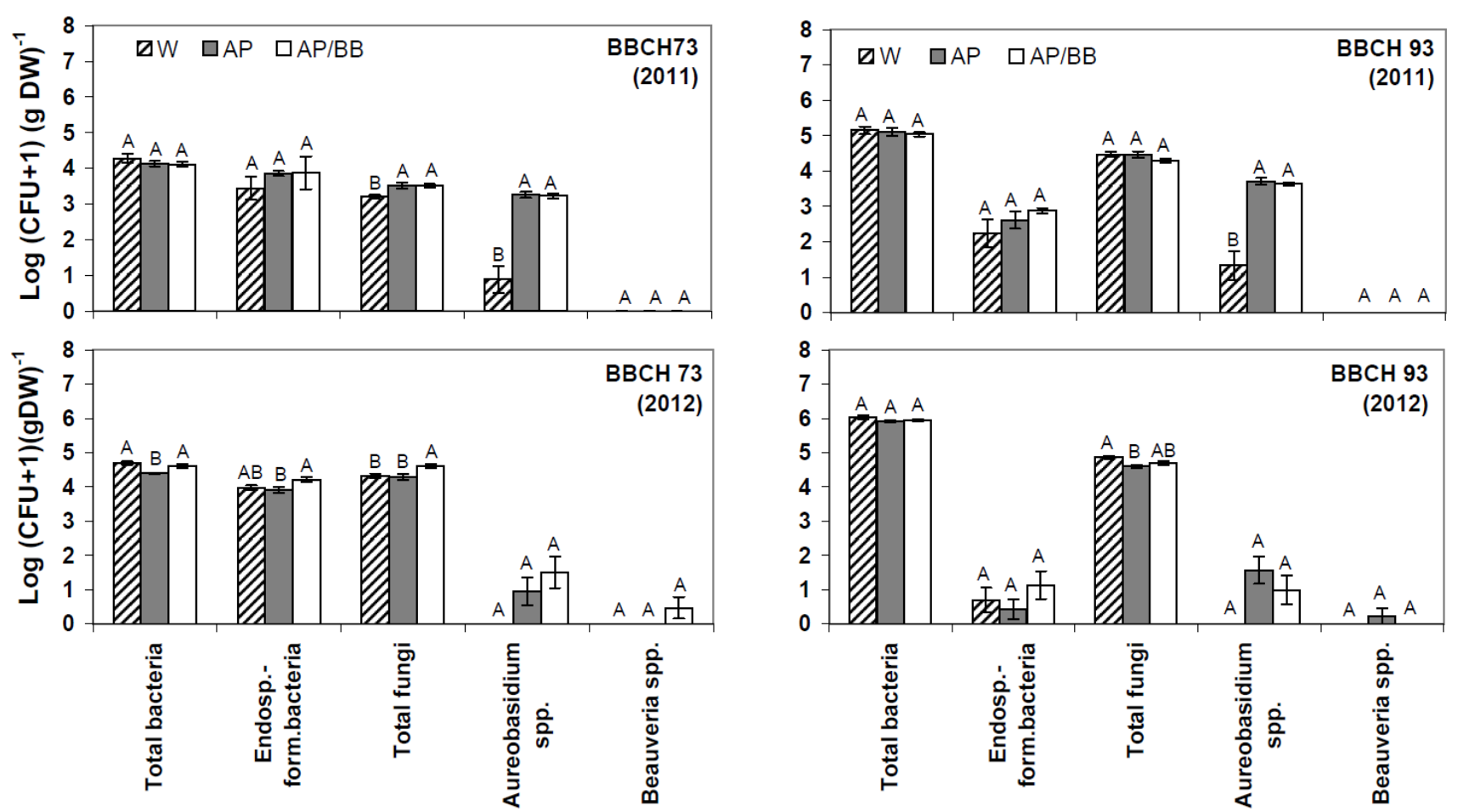

According to 454 pyrosequencing, the composition of the resident leaf microbiome can be described as follows. The resident fungal communities of leaf samples were mainly composed of the orders Filobasidiales, Cystofilobasidiales and Capnodiales in flowering strawberry plants in both years (BBCH 60; Tables 2 and 3), whereas the spectrum of highly abundant fungal orders at this growing stage was extended by Helotiales and Pleosporales in 2012. Cryptococcus (32.9\% to 40.0\%) represented the most abundant fungal genus (Figure 3), followed by Cystofilobasidium, in 2011. The genus Cryptococcus was also most abundant across all untreated leaf samples at this growth stage in 2012. Compared to 2011, the genus Cystofilobasidium was less abundant at BBCH 60 in 2012. 
Table 2. Relative abundance of ITS rRNA reads assigned to fungal orders in the strawberry phyllosphere at three distinct phenological stages (BBCH 60, BBCH 73, and BBCH 93) in 2011 as influenced by treatments with Aureobasidium pullulans as a single strain treatment (AP) or co-inoculated with Beauveria bassiana (AP/BB) through commercial formulations. The control (W) was treated with water from a lotic reservoir.

\begin{tabular}{|c|c|c|c|c|c|c|c|c|c|c|c|c|c|c|c|c|c|c|}
\hline \multirow[b]{3}{*}{ Fungal order } & \multicolumn{6}{|c|}{ BBCH 60 (prior to BCA applications) } & \multicolumn{6}{|c|}{ BBCH 73 (after four BCA applications) } & \multicolumn{6}{|c|}{$\begin{array}{c}\text { BBCH } 93 \text { (four weeks after } \\
\text { last BCA application) }\end{array}$} \\
\hline & \multicolumn{2}{|c|}{$\mathbf{W}^{\mathrm{a}}$} & \multicolumn{2}{|c|}{$\mathbf{A} \mathbf{P}^{\mathrm{a}}$} & \multicolumn{2}{|c|}{$\mathbf{A P} / \mathbf{B B}^{\mathrm{a}}$} & \multicolumn{2}{|c|}{$\mathbf{W}$} & \multicolumn{2}{|c|}{$\mathbf{A P}$} & \multicolumn{2}{|c|}{$\mathbf{A P} / \mathbf{B B}$} & \multicolumn{2}{|c|}{$\mathbf{W}$} & \multicolumn{2}{|c|}{ AP } & \multicolumn{2}{|c|}{ AP/BB } \\
\hline & Mean & SEM & Mean & SEM & Mean & SEM & Mean & SEM & Mean & SEM & Mean & SEM & Mean & SEM & Mean & SEM & Mean & SEM \\
\hline Filobasidiales & 0.336 & 0.021 & 0.329 & 0.025 & 0.400 & 0.026 & 0.088 & 0.013 & 0.088 & 0.023 & 0.050 & 0.009 & 0.389 & 0.026 & 0.270 & 0.036 & 0.311 & 0.021 \\
\hline Dothideales & 0.026 & 0.007 & 0.020 & 0.003 & 0.018 & 0.004 & 0.093 & 0.008 & 0.297 & 0.080 & 0.519 & 0.024 & 0.049 & 0.021 & 0.234 & 0.029 & 0.139 & 0.028 \\
\hline Capnodiales & 0.106 & 0.011 & 0.092 & 0.012 & 0.069 & 0.008 & 0.080 & 0.012 & 0.035 & 0.007 & 0.024 & 0.005 & 0.224 & 0.026 & 0.198 & 0.028 & 0.138 & 0.044 \\
\hline Cystofilobasidiales & 0.162 & 0.020 & 0.176 & 0.027 & 0.166 & 0.032 & 0.016 & 0.002 & 0.009 & 0.003 & 0.007 & 0.004 & 0.044 & 0.046 & 0.011 & 0.003 & 0.006 & 0.002 \\
\hline Pleosporales & 0.033 & 0.006 & 0.035 & 0.008 & 0.027 & 0.001 & 0.096 & 0.010 & 0.103 & 0.035 & 0.040 & 0.004 & 0.047 & 0.020 & 0.076 & 0.022 & 0.114 & 0.034 \\
\hline Sporidiobolales & 0.015 & 0.002 & 0.011 & 0.001 & 0.021 & 0.004 & 0.017 & 0.003 & 0.005 & 0.003 & 0.011 & 0.000 & 0.031 & 0.008 & 0.033 & 0.013 & 0.032 & 0.005 \\
\hline Chaetothyriales & 0.003 & 0.001 & 0.003 & 0.001 & 0.003 & 0.001 & 0.068 & 0.012 & 0.036 & 0.003 & 0.037 & 0.010 & 0.003 & 0.003 & 0.004 & 0.001 & 0.006 & 0.002 \\
\hline Helotiales & 0.011 & 0.004 & 0.008 & 0.002 & 0.006 & 0.001 & 0.010 & 0.001 & 0.004 & 0.002 & 0.020 & 0.006 & 0.053 & 0.021 & 0.017 & 0.008 & 0.041 & 0.007 \\
\hline Hypocreales & 0.014 & 0.001 & 0.032 & 0.013 & 0.007 & 0.001 & 0.011 & 0.004 & 0.010 & 0.002 & 0.008 & 0.003 & 0.020 & 0.012 & 0.013 & 0.006 & 0.016 & 0.008 \\
\hline Tremellales & 0.027 & 0.005 & 0.019 & 0.002 & 0.035 & 0.006 & 0.003 & 0.001 & 0.008 & 0.003 & 0.001 & 0.001 & 0.001 & 0.002 & 0.001 & 0.001 & 0.002 & 0.001 \\
\hline Lecanorales & 0.002 & 0.001 & 0.004 & 0.001 & 0.003 & 0.001 & 0.034 & 0.004 & 0.013 & 0.005 & 0.023 & 0.006 & 0.000 & 0.000 & 0.003 & 0.002 & 0.002 & 0.002 \\
\hline Agaricales & 0.003 & 0.001 & 0.002 & 0.000 & 0.002 & 0.000 & 0.001 & 0.000 & 0.001 & 0.001 & 0.002 & 0.001 & 0.043 & 0.047 & 0.016 & 0.002 & 0.041 & 0.009 \\
\hline Taphrinales & 0.009 & 0.003 & 0.013 & 0.004 & 0.013 & 0.005 & 0.007 & 0.002 & 0.002 & 0.001 & 0.002 & 0.001 & 0.001 & 0.001 & 0.012 & 0.003 & 0.013 & 0.004 \\
\hline Teloschistales & 0.004 & 0.001 & 0.002 & 0.001 & 0.001 & 0.000 & 0.027 & 0.004 & 0.016 & 0.001 & 0.006 & 0.002 & 0.000 & 0.001 & 0.000 & 0.000 & 0.003 & 0.001 \\
\hline Erysiphales & 0.002 & 0.001 & 0.003 & 0.001 & 0.004 & 0.001 & 0.017 & 0.001 & 0.016 & 0.005 & 0.002 & 0.001 & 0.000 & 0.001 & 0.001 & 0.001 & 0.004 & 0.002 \\
\hline Xylariales & 0.007 & 0.002 & 0.002 & 0.000 & 0.003 & 0.001 & 0.008 & 0.002 & 0.011 & 0.007 & 0.009 & 0.002 & 0.000 & 0.000 & 0.002 & 0.001 & 0.000 & 0.000 \\
\hline Pucciniales & 0.000 & 0.000 & 0.001 & 0.000 & 0.000 & 0.000 & 0.017 & 0.003 & 0.010 & 0.005 & 0.009 & 0.004 & 0.000 & 0.000 & 0.000 & 0.000 & 0.000 & 0.000 \\
\hline Others $^{\mathrm{b}}$ & 0.242 & 0.020 & 0.247 & 0.028 & 0.222 & 0.016 & 0.408 & 0.023 & 0.335 & 0.055 & 0.228 & 0.031 & 0.094 & 0.047 & 0.108 & 0.020 & 0.131 & 0.040 \\
\hline
\end{tabular}

${ }^{\mathrm{a}}$ Results from all treatments represent the resident fungal community; ${ }^{\mathrm{b}}$ Contains OTUs accounting for $<1 \%$ of all sequences and OTUs that could not be assigned to orders. 
Table 3. Relative abundance of ITS rRNA reads assigned to fungal orders in the strawberry phyllosphere at three distinct phenological stages (BBCH 60, BBCH 73, and BBCH 93) in 2012 as influenced by treatments with Aureobasidium pullulans as a single strain treatment (AP) or co-inoculated with Beauveria bassiana (AP/BB) through commercial formulations. The control (W) was treated with water from a lotic reservoir.

\begin{tabular}{|c|c|c|c|c|c|c|c|c|c|c|c|c|c|c|c|c|c|c|}
\hline \multirow[b]{3}{*}{ Order } & \multicolumn{6}{|c|}{ BBCH 60 (prior to BCA applications) } & \multicolumn{6}{|c|}{ BBCH 73 (after four BCA applications) } & \multicolumn{6}{|c|}{ BBCH 93 (four weeks after last BCA application) } \\
\hline & \multicolumn{2}{|c|}{$\mathbf{w}^{\mathrm{a}}$} & \multicolumn{2}{|c|}{$\mathbf{A P}^{\mathrm{a}}$} & \multicolumn{2}{|c|}{$\mathbf{A P} / \mathbf{B B}^{\mathrm{a}}$} & \multicolumn{2}{|c|}{$\mathbf{W}$} & \multicolumn{2}{|c|}{ AP } & \multicolumn{2}{|c|}{ AP/BB } & \multicolumn{2}{|c|}{ W } & \multicolumn{2}{|c|}{ AP } & \multicolumn{2}{|c|}{ AP/BB } \\
\hline & Mean & SEM & Mean & SEM & Mean & SEM & Mean & SEM & Mean & SEM & Mean & SEM & Mean & SEM & Mean & SEM & Mean & SEM \\
\hline Filobasidiales & 0.214 & 0.009 & 0.190 & 0.021 & 0.348 & 0.018 & 0.308 & 0.029 & 0.258 & 0.021 & 0.257 & 0.028 & 0.234 & 0.010 & 0.204 & 0.022 & 0.209 & 0.018 \\
\hline Capnodiales & 0.271 & 0.024 & 0.333 & 0.029 & 0.141 & 0.008 & 0.178 & 0.036 & 0.179 & 0.014 & 0.112 & 0.010 & 0.134 & 0.021 & 0.107 & 0.015 & 0.111 & 0.005 \\
\hline Helotiales & 0.100 & 0.009 & 0.119 & 0.022 & 0.063 & 0.012 & 0.059 & 0.008 & 0.102 & 0.027 & 0.047 & 0.014 & 0.267 & 0.008 & 0.360 & 0.052 & 0.234 & 0.035 \\
\hline Pleosporales & 0.087 & 0.013 & 0.066 & 0.010 & 0.066 & 0.010 & 0.063 & 0.004 & 0.083 & 0.006 & 0.042 & 0.010 & 0.066 & 0.014 & 0.053 & 0.006 & 0.064 & 0.007 \\
\hline Cystofilobasidiales & 0.106 & 0.012 & 0.107 & 0.017 & 0.083 & 0.006 & 0.074 & 0.018 & 0.103 & 0.023 & 0.056 & 0.016 & 0.020 & 0.005 & 0.011 & 0.003 & 0.012 & 0.002 \\
\hline Dothideales & 0.016 & 0.005 & 0.017 & 0.003 & 0.023 & 0.005 & 0.025 & 0.001 & 0.015 & 0.005 & 0.338 & 0.020 & 0.005 & 0.002 & 0.043 & 0.003 & 0.077 & 0.013 \\
\hline Sporidiobolales & 0.038 & 0.005 & 0.027 & 0.001 & 0.016 & 0.003 & 0.018 & 0.003 & 0.034 & 0.008 & 0.012 & 0.001 & 0.035 & 0.005 & 0.024 & 0.004 & 0.029 & 0.001 \\
\hline Agaricostilbales & 0.001 & 0.001 & 0.003 & 0.002 & 0.003 & 0.001 & 0.003 & 0.000 & 0.004 & 0.001 & 0.001 & 0.000 & 0.031 & 0.006 & 0.032 & 0.005 & 0.039 & 0.003 \\
\hline Diaporthales & 0.014 & 0.007 & 0.007 & 0.003 & 0.021 & 0.007 & 0.025 & 0.006 & 0.023 & 0.009 & 0.010 & 0.005 & 0.006 & 0.003 & 0.003 & 0.001 & 0.003 & 0.000 \\
\hline Xylariales & 0.006 & 0.003 & 0.011 & 0.004 & 0.014 & 0.003 & 0.026 & 0.009 & 0.015 & 0.004 & 0.011 & 0.006 & 0.002 & 0.001 & 0.001 & 0.001 & 0.001 & 0.000 \\
\hline Hypocreales & 0.009 & 0.004 & 0.013 & 0.003 & 0.018 & 0.006 & 0.013 & 0.005 & 0.012 & 0.005 & 0.006 & 0.002 & 0.002 & 0.000 & 0.002 & 0.000 & 0.002 & 0.001 \\
\hline Taphrinales & 0.004 & 0.003 & 0.003 & 0.001 & 0.009 & 0.001 & 0.005 & 0.001 & 0.004 & 0.001 & 0.003 & 0.000 & 0.014 & 0.002 & 0.012 & 0.002 & 0.018 & 0.003 \\
\hline Agaricales & 0.000 & 0.000 & 0.001 & 0.000 & 0.013 & 0.003 & 0.011 & 0.002 & 0.004 & 0.003 & 0.004 & 0.001 & 0.006 & 0.001 & 0.009 & 0.006 & 0.008 & 0.002 \\
\hline Others ${ }^{b}$ & 0.133 & 0.002 & 0.103 & 0.001 & 0.184 & 0.001 & 0.193 & 0.002 & 0.164 & 0.001 & 0.101 & 0.000 & 0.178 & 0.001 & 0.139 & 0.000 & 0.193 & 0.001 \\
\hline
\end{tabular}

${ }^{\mathrm{a}}$ Results from all treatments represent the resident fungal community; ${ }^{\mathrm{b}}$ Contains OTUs accounting for $<1 \%$ of all sequences and OTUs that could not be assigned to orders. 
Figure 3. Most abundant fungal genera in the strawberry phyllosphere at three distinct phenological stages $(\mathrm{BBCH} 60, \mathrm{BBCH} 73$, and $\mathrm{BBCH} 93)$ during two subsequent years $(2011,2012)$ as influenced by treatments with Aureobasidium pullulans as a single strain treatment (AP) or co-inoculated with Beauveria bassiana (AP/BB) and as determined by 454 pyrosequencing. The control (W) was treated with water from a lotic reservoir. Results from all treatments at $\mathrm{BBCH} 60$ (prior to $\mathrm{BCA}$ applications) represent the resident fungal community. The color legend refers to the relative abundance ( $\%$ of total sequences) of fungal OTUs.

\begin{tabular}{|c|c|c|c|c|c|c|c|c|c|c|c|c|c|c|c|c|c|c|}
\hline \multirow[b]{3}{*}{ Genus } & \multicolumn{9}{|c|}{2011} & \multicolumn{9}{|c|}{2012} \\
\hline & \multicolumn{3}{|c|}{$\mathrm{BBCH} 60$} & \multicolumn{3}{|c|}{$\mathrm{BBCH} 73$} & \multicolumn{3}{|c|}{$\mathrm{BBCH} 93$} & \multicolumn{3}{|c|}{$\mathrm{BBCH} 60$} & \multicolumn{3}{|c|}{$\mathrm{BBCH} 73$} & \multicolumn{3}{|c|}{$\mathrm{BBCH} 93$} \\
\hline & 3 & $\frac{0}{4}$ & $\frac{\frac{m}{\underline{m}}}{\frac{\alpha}{\alpha}}$ & 3 & $\frac{0}{<}$ & $\frac{\frac{m}{\frac{m}{\alpha}}}{\frac{\alpha}{\alpha}}$ & 3 & $\frac{0}{4}$ & $\frac{\stackrel{m}{\frac{m}{\alpha}}}{\frac{\alpha}{\alpha}}$ & 3 & $\frac{0}{4}$ & $\frac{\frac{m}{m}}{\frac{\underline{\alpha}}{\alpha}}$ & 3 & $\frac{0}{4}$ & $\frac{\frac{m}{\frac{m}{\alpha}}}{\frac{\alpha}{\alpha}}$ & 3 & $\frac{0}{4}$ & 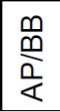 \\
\hline \multicolumn{19}{|l|}{ Cryptococcus } \\
\hline \multicolumn{19}{|c|}{ Aureobasidium } \\
\hline \multicolumn{19}{|c|}{ Cystofilobasidium } \\
\hline \multicolumn{19}{|c|}{ Davidiella } \\
\hline \multirow{2}{*}{\multicolumn{19}{|c|}{ Cladosporium }} \\
\hline \multicolumn{17}{|l|}{ Alternaria } & & \\
\hline \multirow{2}{*}{\multicolumn{19}{|c|}{$\begin{array}{l}\text { Epicoccum } \\
\text { Verticillium }\end{array}$}} \\
\hline & & & & & & & & & & & & & & & & & & \\
\hline \multicolumn{19}{|c|}{ Rhodotorula } \\
\hline \multicolumn{19}{|c|}{ Sporobolomyces } \\
\hline \multicolumn{19}{|c|}{ Lewia } \\
\hline \multirow{2}{*}{\multicolumn{19}{|c|}{ Fusarium }} \\
\hline & & & & & & & & & & & & & & & & & & \\
\hline Pucciniastrum & & & & & & & & & & & & & & & & & & \\
\hline \multicolumn{19}{|l|}{ Udeniomyces } \\
\hline \multirow{2}{*}{\multicolumn{19}{|c|}{ Physcia }} \\
\hline & & & & & & & & & & & & & \multicolumn{6}{|c|}{ Blumeria } \\
\hline Phoma & & & & & & & & & & & & & & & & & & \\
\hline Bullera & & & & & & & & & & & & & & & & & & \\
\hline Phaeophyscia & & & & & & & & & & & & & & & & & & \\
\hline Parasola & & & & & & & & & & & & & & & & & & \\
\hline Sporormia & & & & & & & & & & & & & & & & & & \\
\hline Cordyceps & & & & & & & & & & & & & & & & & & \\
\hline Truncatella & & & & & & & & & & & & & & & & & & \\
\hline Pilidium & & & & & & & & & & & & & & & & & & \\
\hline Gnomoniopsi & & & & & & & & & & & & & & & & & & \\
\hline Cadophora & & & & & & & & & & & & & & & & & & \\
\hline Ramularia & & & & & & & & & & & & & & & & & & \\
\hline Bensingtonia & & & & & & & & & & & & & & & & & & \\
\hline
\end{tabular}

The composition of fungal communities did not differ between the untreated leaf samples in flowering strawberry plants in 2011 (ANOSIM: $p=0.255 ; \alpha=0.05 ; R=0.07$ ). In contrast, in 2012 the composition of fungal communities in leaf samples obtained from plots intended for multiple strain treatments significantly differed from leaf samples from plots designated for treatments with water ( $p=0.0316 ; \alpha=0.05 ; R=1)$ and A. pullulans $(p=0.0304 ; \alpha=0.05 ; R=1)$, respectively. These contrasting results from the two years at the early phenological stage is also pronounced by diversity index (Shannon diversity indices) and genus richness (Chao1), which were similar between all plots in 2011 (Figure 4A,C) but displayed a significantly higher fungal richness in leaf samples obtained from 
plots intended for the co-inoculation treatment than in the other leaf samples collected from flowering strawberry plants in 2012 (Figure 4D). Overall diversity of fungal communities, however, was similar among all leaf samples in 2012.

Figure 4. Shannon diversity indices and Chaol estimators describing the biodiversity and taxa richness of fungal phyllosphere communities at three distinct phenological stages $(\mathrm{BBCH}$ 60, $\mathrm{BBCH}$ 73, and $\mathrm{BBCH}$ 93) as influenced by treatments with Aureobasidium pullulans as a single strain treatment (AP) or co-inoculated with Beauveria bassiana (AP/BB) in 2011 (A,C) and 2012 (B,D). The control (W) was treated with water from a lotic reservoir. Error bars represent SEM values. Columns marked with the same letters are not significantly different within each phenological stage $(\alpha=0.05)$.
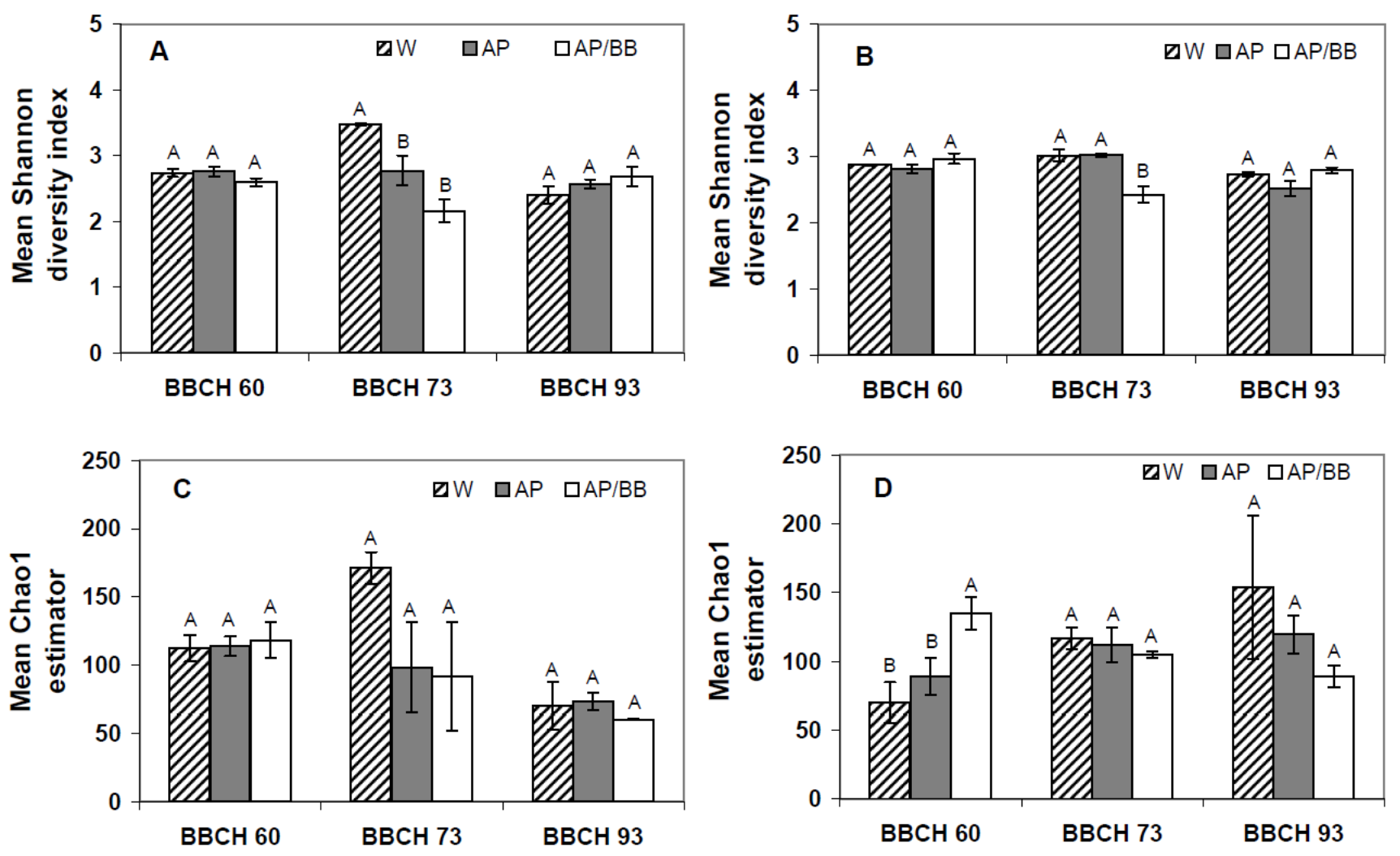

The resident leaf microbiome of fruit-setting plants was dominated by the fungal orders Filobasidiales, Dothideales, Capnodiales and Pleosporales in 2011 (Table 2). In these samples, the abundance of Cryptococcus decreased to $8.7 \%$, while the genus Aureobasidium became similarly abundant (Figure 3). In 2012, the fungal orders Filobasidiales and Capnodiales together accounted for $48.6 \%$ of the total resident fungal community on leaves collected from fruit-setting strawberries in the control samples (Table 3). At this stage in 2012, the genus Cryptococcus was the major genus in the control samples. When old dying leaves were scrutinized in 2011, the resident fungal community was dominated by the orders Filobasidiales and Capnodiales in the control samples (Table 2), while also a third order, the Helotiales, was highly represented at this stage in 2012 (Table 3). The abundance of Cryptococcus made up 38.3\% (2011) and 23.3\% (2012) in the non-treated leaf samples (Figure 3). Thus, in both years, the genus Cryptococcus was highly abundant in the phyllosphere of strawberries throughout the season. This yeast is well adapted to the phyllosphere and is commonly isolated from 
the phyllosphere of different crops [20,29,30] as well as from ripe strawberry fruit [31]. Likewise, the yeast-like fungus $A$. pullulans is considered as an ubiquitous and well adapted resident of the phyllosphere [28,32]. The absence of Aureobasidium spp. in plate counts from untreated leaf samples at later phenological stages in 2012 was, therefore, unexpected.

The resident bacterial phyllosphere communities of flowering plants were essentially composed of the orders Sphingobacteriales, Burkholderiales and Actinomycetales in 2011 (Table 4), whereas the Rickettsiales and Actinomycetales represented the most abundant orders at this stage in 2012 (Table 5). The bacterial genus Hymenobacter was a dominant bacterial resident in the phyllosphere during flowering (BBCH 60) in 2011, but displayed low abundance in 2012 (Figure 5). Although the occurrence of the genus Hymenobacter has been reported from the plant's phyllosphere [33,34], there is still little knowledge of its role in the phyllosphere. Furthermore, the relative abundance of the genus Bacillus was high in leaf samples from plots designated for later multiple strain treatments (15.8\%) in flowering plants in 2012, while it was below $4.0 \%$ for the other leaf samples. As a consequence, diversity and genus richness as well as the composition of bacterial communities (ANOSIM: $p=0.065 ; \alpha=0.05 ; R=0.325$ ) were similar among all untreated leaf samples during flowering in 2011 (Figure 6A,C), whereas the bacterial composition in leaf samples from plots designated for multiple strain treatments significantly differed from those designated for water (ANOSIM: $p=0.030$; $\alpha=0.05 ; R=0.771$ ) and single treatments with $A$. pullulans (ANOSIM: $p=0.029 ; \alpha=0.05$; $R=0.833$ ) at this phenological stage in 2012. Diversity as well as genus richness, though, was similar between the untreated leaf samples collected during flowering in 2012 (Figure 6B,D).

The resident leaf microbiome was mainly composed of the Enterobacteriales and Burkholderiales during fruit-setting (BBCH 73) in 2012 (Table 5) with Yersinia, Microcoleus and Pseudomonas as the most abundant bacterial genera (Figure 5). The orders Enterobacteriales, Sphingomonadales and Pseudomonadales were highly abundant in leaf samples from control plots at BBCH 93 in 2011 (Table 4). Accordingly, the genera Sphingomonas and Pseudomonas were predominant in these leaf samples, whereas the abundance of the genus Hymenobacter decreased to $2.4 \%$ (Figure 5). The bacterial order Enterobacteriales was predominant in control leaf samples at BBCH 93 in 2012, followed by the Sphingomonadales and Burkholderiales (Table 5). The genera Erwinia, Sphingomonas and Pseudomonas together accounted for about $44 \%$ of the total resident bacterial community in the control samples at this sampling date. 
Table 4. Relative abundance of $16 \mathrm{~S}$ rRNA reads assigned to bacterial orders in the strawberry phyllosphere at two distinct phenological stages $(\mathrm{BBCH} 60$ and $\mathrm{BBCH} 93)$ in 2011 as influenced by treatments with Aureobasidium pullulans as a single strain treatment (AP) or co-inoculated with Beauveria bassiana (AP/BB) through commercial formulations. The control (W) was treated with water from a lotic reservoir.

\begin{tabular}{|c|c|c|c|c|c|c|c|c|c|c|c|c|}
\hline \multirow[b]{3}{*}{ Bacterial order } & \multicolumn{6}{|c|}{ BBCH 60 (prior to BCA applications) } & \multicolumn{6}{|c|}{ BBCH 93 (four weeks after last BCA application) } \\
\hline & \multicolumn{2}{|c|}{$\mathbf{w}^{\mathrm{a}}$} & \multicolumn{2}{|c|}{$\mathbf{A P}{ }^{\mathrm{a}}$} & \multicolumn{2}{|c|}{$\mathbf{A P} / \mathbf{B B}^{a}$} & \multicolumn{2}{|c|}{$\mathbf{W}$} & \multicolumn{2}{|c|}{ AP } & \multicolumn{2}{|c|}{$\mathbf{A P} / \mathbf{B B}$} \\
\hline & Mean & SEM & Mean & SEM & Mean & SEM & Mean & SEM & Mean & SEM & Mean & SEM \\
\hline Burkholderiales & 0.183 & 0.052 & 0.153 & 0.072 & 0.092 & 0.015 & 0.074 & 0.037 & 0.144 & 0.040 & 0.130 & 0.031 \\
\hline Sphingobacteriales & 0.213 & 0.050 & 0.200 & 0.046 & 0.167 & 0.002 & 0.017 & 0.017 & 0.112 & 0.056 & 0.053 & 0.017 \\
\hline Actinomycetales & 0.130 & 0.010 & 0.071 & 0.014 & 0.160 & 0.010 & 0.057 & 0.033 & 0.044 & 0.018 & 0.102 & 0.023 \\
\hline Sphingomonadales & 0.058 & 0.029 & 0.041 & 0.016 & 0.097 & 0.026 & 0.220 & 0.022 & 0.106 & 0.028 & 0.162 & 0.037 \\
\hline Rickettsiales & 0.049 & 0.005 & 0.089 & 0.046 & 0.084 & 0.038 & 0.007 & 0.007 & 0.043 & 0.034 & 0.028 & 0.012 \\
\hline Pseudomonadales & 0.006 & 0.002 & 0.000 & 0.000 & 0.050 & 0.043 & 0.189 & 0.129 & 0.169 & 0.029 & 0.156 & 0.044 \\
\hline Enterobacteriales & 0.010 & 0.008 & 0.002 & 0.002 & 0.000 & 0.000 & 0.229 & 0.092 & 0.158 & 0.069 & 0.089 & 0.019 \\
\hline Rhizobiales & 0.033 & 0.008 & 0.015 & 0.001 & 0.029 & 0.014 & 0.056 & 0.021 & 0.090 & 0.008 & 0.107 & 0.024 \\
\hline Lactobacillales & 0.002 & 0.002 & 0.114 & 0.114 & 0.026 & 0.010 & 0.015 & 0.008 & 0.023 & 0.011 & 0.033 & 0.014 \\
\hline Rhodospirillales & 0.021 & 0.008 & 0.024 & 0.005 & 0.026 & 0.010 & 0.020 & 0.018 & 0.004 & 0.004 & 0.027 & 0.008 \\
\hline Bacillales & 0.033 & 0.011 & 0.012 & 0.001 & 0.033 & 0.017 & 0.007 & 0.006 & 0.019 & 0.019 & 0.011 & 0.004 \\
\hline Pasteurellales & 0.002 & 0.002 & 0.071 & 0.056 & 0.000 & 0.000 & 0.000 & 0.000 & 0.002 & 0.002 & 0.009 & 0.007 \\
\hline Exiguobacterales & 0.090 & 0.090 & 0.009 & 0.002 & 0.011 & 0.004 & 0.000 & 0.000 & 0.000 & 0.000 & 0.000 & 0.000 \\
\hline Rhodobacterales & 0.004 & 0.002 & 0.005 & 0.005 & 0.026 & 0.005 & 0.008 & 0.006 & 0.012 & 0.012 & 0.003 & 0.003 \\
\hline Myxococcales & 0.005 & 0.003 & 0.002 & 0.001 & 0.015 & 0.001 & 0.001 & 0.001 & 0.008 & 0.006 & 0.005 & 0.003 \\
\hline Chroococcales & 0.014 & 0.008 & 0.024 & 0.021 & 0.011 & 0.011 & 0.001 & 0.001 & 0.000 & 0.000 & 0.000 & 0.000 \\
\hline Bifidobacteriales & 0.001 & 0.001 & 0.034 & 0.032 & 0.004 & 0.004 & 0.000 & 0.000 & 0.002 & 0.002 & 0.007 & 0.007 \\
\hline Neisseriales & 0.003 & 0.002 & 0.039 & 0.036 & 0.004 & 0.004 & 0.000 & 0.000 & 0.002 & 0.002 & 0.002 & 0.002 \\
\hline Bacteroidales & 0.000 & 0.000 & 0.000 & 0.000 & 0.000 & 0.000 & 0.059 & 0.059 & 0.000 & 0.000 & 0.000 & 0.000 \\
\hline Flavobacteriales & 0.000 & 0.000 & 0.001 & 0.001 & 0.004 & 0.004 & 0.007 & 0.006 & 0.017 & 0.017 & 0.002 & 0.002 \\
\hline Xanthomonadales & 0.006 & 0.003 & 0.002 & 0.002 & 0.011 & 0.004 & 0.001 & 0.001 & 0.007 & 0.006 & 0.001 & 0.001 \\
\hline Solirubrobacterales & 0.006 & 0.004 & 0.007 & 0.003 & 0.008 & 0.008 & 0.001 & 0.001 & 0.000 & 0.000 & 0.000 & 0.000 \\
\hline iii1-15 & 0.003 & 0.002 & 0.002 & 0.001 & 0.019 & 0.012 & 0.001 & 0.001 & 0.000 & 0.000 & 0.003 & 0.003 \\
\hline Acidimicrobiales & 0.003 & 0.002 & 0.001 & 0.001 & 0.008 & 0.008 & 0.001 & 0.001 & 0.002 & 0.002 & 0.005 & 0.003 \\
\hline 0319-7L14 & 0.003 & 0.002 & 0.000 & 0.000 & 0.011 & 0.004 & 0.000 & 0.000 & 0.000 & 0.000 & 0.001 & 0.001 \\
\hline Others ${ }^{b}$ & 0.123 & 0.040 & 0.080 & 0.016 & 0.107 & 0.061 & 0.030 & 0.020 & 0.037 & 0.030 & 0.062 & 0.037 \\
\hline
\end{tabular}

${ }^{\mathrm{a}}$ Results from all treatments represent the resident bacterial community; ${ }^{\mathrm{b}}$ Contains OTUs accounting for $<1 \%$ of all sequences and OTUs that could not be assigned to orders; Data for BBCH 73 (after four BCA applications) in 2011 were insufficient and are not shown. 
Table 5. Relative abundance of $16 \mathrm{~S}$ rRNA reads assigned to bacterial orders in the strawberry phyllosphere at three distinct phenological stages (BBCH 60, BBCH 73, and BBCH 93) in 2012 as influenced by treatments with Aureobasidium pullulans as a single strain treatment (AP) or co-inoculated with Beauveria bassiana (AP/BB) through commercial formulations. The control (W) was treated with water from a lotic reservoir.

\begin{tabular}{|c|c|c|c|c|c|c|c|c|c|c|c|c|c|c|c|c|c|c|}
\hline \multirow[b]{3}{*}{ Bacterial order } & \multicolumn{6}{|c|}{ BBCH 60 (prior to BCA applications) } & \multicolumn{6}{|c|}{ BBCH 73 (after four BCA applications) } & \multicolumn{6}{|c|}{ BBCH 93 (four weeks after last BCA application) } \\
\hline & \multicolumn{2}{|c|}{$\mathbf{W}^{\mathrm{a}}$} & \multicolumn{2}{|c|}{$\mathbf{A P}^{\mathrm{a}}$} & \multicolumn{2}{|c|}{$\mathbf{A P} / \mathbf{B B}^{\mathrm{a}}$} & \multicolumn{2}{|c|}{ W } & \multicolumn{2}{|c|}{$\mathbf{A P}$} & \multicolumn{2}{|c|}{ AP/BB } & \multicolumn{2}{|c|}{ W } & \multicolumn{2}{|c|}{ AP } & \multicolumn{2}{|c|}{ AP/BB } \\
\hline & Mean & SEM & Mean & SEM & Mean & SEM & Mean & SEM & Mean & SEM & Mean & SEM & Mean & SEM & Mean & SEM & Mean & SEM \\
\hline Sphingomonadales & 0.039 & 0.004 & 0.034 & 0.010 & 0.087 & 0.029 & 0.070 & 0.016 & 0.037 & 0.009 & 0.122 & 0.082 & 0.182 & 0.028 & 0.223 & 0.017 & 0.314 & 0.052 \\
\hline Enterobacteriales & 0.034 & 0.021 & 0.005 & 0.001 & 0.091 & 0.043 & 0.151 & 0.099 & 0.009 & 0.004 & 0.012 & 0.008 & 0.385 & 0.082 & 0.169 & 0.034 & 0.164 & 0.059 \\
\hline Burkholderiales & 0.057 & 0.010 & 0.070 & 0.011 & 0.103 & 0.009 & 0.111 & 0.014 & 0.080 & 0.015 & 0.172 & 0.023 & 0.125 & 0.021 & 0.110 & 0.013 & 0.130 & 0.015 \\
\hline Rickettsiales & 0.194 & 0.037 & 0.173 & 0.017 & 0.053 & 0.023 & 0.084 & 0.041 & 0.186 & 0.050 & 0.106 & 0.019 & 0.012 & 0.005 & 0.012 & 0.006 & 0.026 & 0.013 \\
\hline Sphingobacteriales & 0.045 & 0.006 & 0.077 & 0.018 & 0.096 & 0.016 & 0.068 & 0.016 & 0.097 & 0.031 & 0.188 & 0.002 & 0.053 & 0.011 & 0.093 & 0.018 & 0.077 & 0.027 \\
\hline Rhizobiales & 0.069 & 0.012 & 0.067 & 0.007 & 0.083 & 0.023 & 0.045 & 0.009 & 0.050 & 0.006 & 0.031 & 0.001 & 0.090 & 0.017 & 0.132 & 0.008 & 0.120 & 0.012 \\
\hline Actinomycetales & 0.157 & 0.015 & 0.123 & 0.014 & 0.062 & 0.012 & 0.079 & 0.021 & 0.138 & 0.014 & 0.048 & 0.024 & 0.018 & 0.004 & 0.018 & 0.004 & 0.023 & 0.006 \\
\hline Pseudomonadales & 0.020 & 0.008 & 0.014 & 0.003 & 0.031 & 0.009 & 0.084 & 0.072 & 0.037 & 0.015 & 0.032 & 0.004 & 0.084 & 0.040 & 0.188 & 0.034 & 0.109 & 0.040 \\
\hline Chroococcales & 0.051 & 0.014 & 0.024 & 0.013 & 0.045 & 0.012 & 0.073 & 0.025 & 0.074 & 0.040 & 0.116 & 0.094 & 0.001 & 0.001 & 0.000 & 0.000 & 0.000 & 0.000 \\
\hline Bacillales & 0.040 & 0.009 & 0.029 & 0.009 & 0.170 & 0.012 & 0.029 & 0.007 & 0.033 & 0.008 & 0.025 & 0.016 & 0.001 & 0.001 & 0.000 & 0.000 & 0.001 & 0.001 \\
\hline Rhodospirillales & 0.042 & 0.010 & 0.037 & 0.000 & 0.025 & 0.005 & 0.034 & 0.010 & 0.031 & 0.007 & 0.028 & 0.000 & 0.002 & 0.001 & 0.001 & 0.001 & 0.001 & 0.001 \\
\hline Xanthomonadales & 0.022 & 0.004 & 0.019 & 0.010 & 0.010 & 0.004 & 0.010 & 0.004 & 0.017 & 0.006 & 0.012 & 0.008 & 0.001 & 0.001 & 0.003 & 0.001 & 0.004 & 0.003 \\
\hline iii1-15 & 0.010 & 0.002 & 0.034 & 0.006 & 0.006 & 0.001 & 0.011 & 0.005 & 0.014 & 0.006 & 0.004 & 0.004 & 0.000 & 0.000 & 0.000 & 0.000 & 0.000 & 0.000 \\
\hline Rhodobacterales & 0.005 & 0.003 & 0.007 & 0.004 & 0.006 & 0.003 & 0.002 & 0.001 & 0.006 & 0.003 & 0.000 & 0.000 & 0.009 & 0.004 & 0.012 & 0.004 & 0.009 & 0.002 \\
\hline Myxococcales & 0.006 & 0.003 & 0.014 & 0.005 & 0.008 & 0.003 & 0.008 & 0.003 & 0.010 & 0.004 & 0.007 & 0.001 & 0.000 & 0.000 & 0.000 & 0.000 & 0.000 & 0.000 \\
\hline Solirubrobacterales & 0.019 & 0.005 & 0.014 & 0.002 & 0.005 & 0.002 & 0.009 & 0.003 & 0.004 & 0.002 & 0.002 & 0.002 & 0.000 & 0.000 & 0.000 & 0.000 & 0.000 & 0.000 \\
\hline Acidimicrobiales & 0.016 & 0.006 & 0.011 & 0.003 & 0.006 & 0.001 & 0.007 & 0.003 & 0.011 & 0.002 & 0.000 & 0.000 & 0.000 & 0.000 & 0.000 & 0.000 & 0.000 & 0.000 \\
\hline Gaiellales & 0.013 & 0.006 & 0.011 & 0.005 & 0.007 & 0.001 & 0.006 & 0.003 & 0.010 & 0.005 & 0.003 & 0.003 & 0.000 & 0.000 & 0.000 & 0.000 & 0.000 & 0.000 \\
\hline Oceanospirillales & 0.020 & 0.020 & 0.000 & 0.000 & 0.001 & 0.000 & 0.001 & 0.001 & 0.018 & 0.018 & 0.000 & 0.000 & 0.000 & 0.000 & 0.000 & 0.000 & 0.000 & 0.000 \\
\hline Flavobacteriales & 0.003 & 0.001 & 0.013 & 0.008 & 0.002 & 0.001 & 0.002 & 0.001 & 0.002 & 0.001 & 0.002 & 0.002 & 0.001 & 0.001 & 0.003 & 0.001 & 0.002 & 0.001 \\
\hline Chthoniobacterales & 0.002 & 0.002 & 0.011 & 0.003 & 0.001 & 0.000 & 0.001 & 0.001 & 0.000 & 0.000 & 0.000 & 0.000 & 0.000 & 0.000 & 0.000 & 0.000 & 0.000 & 0.000 \\
\hline Others $^{\mathrm{b}}$ & 0.138 & 0.050 & 0.210 & 0.072 & 0.102 & 0.022 & 0.120 & 0.044 & 0.135 & 0.030 & 0.090 & 0.023 & 0.037 & 0.020 & 0.036 & 0.010 & 0.022 & 0.005 \\
\hline
\end{tabular}

${ }^{\mathrm{a}}$ Results from all treatments represent the resident bacterial community; ${ }^{\mathrm{b}}$ Contains OTUs accounting for $<1 \%$ of all sequences and OTUs that could not be assigned to orders. 
Figure 5. Most abundant bacterial genera in the strawberry phyllosphere at three distinct phenological stages $(\mathrm{BBCH} 60, \mathrm{BBCH} 73$, and $\mathrm{BBCH}$ 93) during two subsequent years $(2011,2012)$ as influenced by treatments with Aureobasidium pullulans as a single strain treatment (AP) or co-inoculated with Beauveria bassiana (AP/BB) and as determined by 454 pyrosequencing. The control (W) was treated with water from a lotic reservoir. Results from all treatments at $\mathrm{BBCH} 60$ (prior to $\mathrm{BCA}$ applications) represent the resident bacterial community. Data for the second sampling (BBCH 73, after four BCA applications) in 2011 were insufficient and are not shown. The color legend refers to the relative abundance ( $\%$ of total sequences) of bacterial OTUs.

\begin{tabular}{|c|c|c|c|c|c|c|c|c|c|c|c|c|c|c|}
\hline \multirow[b]{3}{*}{ Genus } & \multicolumn{6}{|c|}{2011} & \multicolumn{8}{|c|}{2012} \\
\hline & \multicolumn{3}{|c|}{$\mathrm{BBCH} 60$} & \multicolumn{3}{|c|}{$\mathrm{BBCH} 93$} & \multicolumn{3}{|c|}{$\mathrm{BBCH} 60$} & \multicolumn{2}{|c|}{$\mathrm{BBCH} 73$} & \multicolumn{3}{|c|}{$\mathrm{BBCH} 93$} \\
\hline & 3 & 是 & $\begin{array}{l}\frac{m}{m} \\
\frac{\underline{\alpha}}{\alpha}\end{array}$ & 3 & $\frac{0}{<}$ & $\begin{array}{l}\frac{m}{n} \\
\frac{\alpha}{\alpha}\end{array}$ & 3 & $\frac{0}{4}$ & $\frac{m}{\frac{m}{\alpha}}$ & $\frac{0}{<}$ & $\begin{array}{l}\frac{m}{n} \\
\frac{\alpha}{\alpha}\end{array}$ & 3 & $\frac{0}{4}$ & 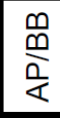 \\
\hline \multicolumn{15}{|l|}{ Hymenobacter } \\
\hline \multicolumn{15}{|l|}{ Sphingomonas } \\
\hline \multirow{2}{*}{\multicolumn{15}{|c|}{$\begin{array}{l}\text { Pseudomonas } \\
\text { Lactobacillus }\end{array}$}} \\
\hline & & & & & \multicolumn{10}{|c|}{ Lactobacillus } \\
\hline \multicolumn{15}{|l|}{ Erwinia } \\
\hline \multicolumn{15}{|l|}{ Variovorax } \\
\hline \multicolumn{15}{|l|}{ Methylobacterium } \\
\hline \multicolumn{15}{|l|}{ Exiguobacterium } \\
\hline \multicolumn{15}{|l|}{ Skermanella } \\
\hline \multicolumn{15}{|l|}{ Janthinobacterium } \\
\hline \multicolumn{15}{|l|}{ Bacillus } \\
\hline \multicolumn{15}{|l|}{ Bifidobacterium } \\
\hline \multicolumn{15}{|l|}{ Dysgonomonas } \\
\hline Dyadobacter & & & & & & & & & & & & & & \\
\hline Rathayibacter & & & & & & & & & & & & & & \\
\hline Acidovorax & & & & & & & & & & & & & & \\
\hline Paracoccus & & & & & & & & & & & & & & \\
\hline Microcoleus & & & & & & & & & & & & & & \\
\hline Agrobacterium & & & & & & & & & & & & & & \\
\hline Burkholderia & & & & & & & & & & & & & & \\
\hline Balneimonas & & & & & & & & & & & & & & \\
\hline Pedobacter & & & & & & & & & & & & & & \\
\hline Kaistobacter & & & & & & & & & & & & & & \\
\hline Chryseobacterium & & & & & & & & & & & & & & \\
\hline Flavobacterium & & & & & & & & & & & & & & \\
\hline Steroidobacter & & & & & & & & & & & & & & \\
\hline Cellulomonas & & & & & & & & & & & & & & \\
\hline Enterococcus & & & & & & & & & & & & & & \\
\hline Citrobacter & & & & & & & & & & & & & & \\
\hline Yersinia & & & & & & & & & & & & & & \\
\hline Balneimonas & & & & & & & & & & & & & & \\
\hline A4 & & & & & & & & & & & & & & \\
\hline Agrobacterium & & & & & & & & & & & & & & \\
\hline Paenibacillus & & & & & & & & & & & & & & \\
\hline Steroidobacter & & & & & & & & & & & & & & \\
\hline Candidatus Portiera & & & & & & & & & & & & & & \\
\hline Buchnera & & & & & & & & & & & & & & \\
\hline Kaistobacter & & & & & & & & & & & & & & \\
\hline Gluconacetobacter & & & & & & & & & & & & & & \\
\hline
\end{tabular}


Figure 6. Shannon diversity indices and Chaol estimators describing the biodiversity and taxa richness of bacterial phyllosphere communities at three distinct phenological stages $(\mathrm{BBCH}$ 60, $\mathrm{BBCH}$ 73, and $\mathrm{BBCH}$ 93) as influenced by treatments with Aureobasidium pullulans as a single treatment (AP) or co-inoculated with Beauveria bassiana (AP/BB) in 2011 (A,C) and 2012 (B,D). The control (W) was treated with water from a lotic reservoir. Error bars represent SEM values. Columns marked with the same letters are not significantly different within each phenological stage $(\alpha=0.05)$.
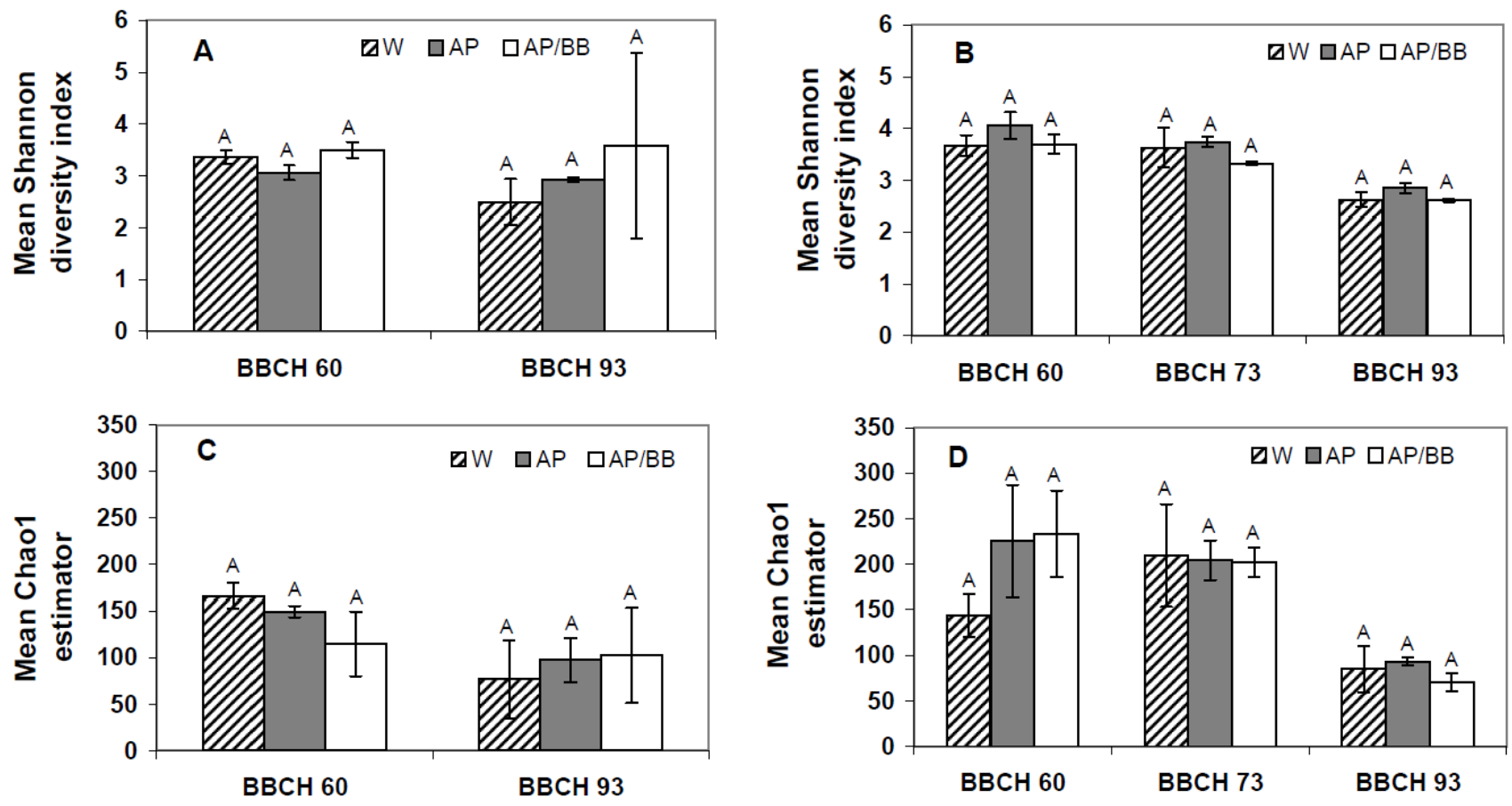

The results from both plate counts and 454 pyrosequencing suggest a general change in the resident leaf microbiome during the course of the growing season of strawberries. Seasonal changes of the microbial communities in the phyllosphere of strawberries have previously been observed [22]. It is suggested that the changes in microbial phyllosphere communities are driven by changing environmental conditions in general (e.g., permanent increase of day length, temperature, and irradiation) and by environmental events such as heavy rainfall $[9,18]$. In addition, the ageing of leaves also contributes to seasonal changes in microbial phyllosphere communities as already shown for the phyllosphere of sugar beet [29], mango [20], and cottonwood trees [35]. Leaf age affects leaf properties, both with respect to the leaf morphology [19] and with respect to physiological properties, such as photosynthetic activity [36]. These changes affect the size of the organic compound pool, sites of exudation and the quality of organic compounds exudated over the cuticle and, in turn, affect the microbial community composition. In the present study, the number of resident fungi was considerably increased at the end of the growing season both in 2011 and 2012, which most probably resulted from a general increase in nutrient leakage in ageing or dying leaves [18].

In addition to seasonal changes, considerable differences in culturable and non-culturable microbial communities were observed between 2011 and 2012. The difference between 2011 and 2012 was most pronounced for bacterial communities as determined by 454 pyrosequencing. 


\subsection{Effects of Introducing BCAs to the Strawberry Phyllosphere}

According to plate counts, Aureobasidium spp. were significantly increased in BCA-treated leaf samples at fruit-setting stage $(\mathrm{BBCH} 73)$, i.e., after four $\mathrm{BCA}$ applications and when old leaves were dying (BBCH 93), i.e., four weeks after adjournment of BCA applications in 2011 (Figure 2). In contrast, Aureobasidium counts were neither significantly elevated in BCA-treated leaf samples at $\mathrm{BBCH} 73$ nor at $\mathrm{BBCH} 93$ in 2012 as compared to the controls.

In addition, according to 454 pyrosequencing, the relative abundance of Aureobasidium was significantly higher in $A$. pullulans-treated leaf samples than in control samples $(p=0.024)$ at $\mathrm{BBCH}$ 73 in 2011. At this stage, leaf samples from plots treated with multiple strains not only showed significantly increased abundances of Aureobasidium as compared to control leaf samples $(p<0.001)$ but also as compared to single strain treatments with $A$. pullulans ( $p=0.022$, Figure 7$)$. In 2012, the relative abundance of Aureobasidium was only significantly increased in co-inoculated leaf samples during fruit-setting as compared to control $(p<0.001)$ and A. pullulans-treated leaf samples $(p<0.001)$. Four weeks after the last BCA application (BBCH 93), pronounced effects, in terms of relative abundance of Aureobasidium spp., were observed during the first experimental year, whereas its relative abundance was comparably low $(<8 \%)$ in all leaf samples in the second year (Figure 7).

Both, plate counts as well as 454 pyrosequencing results indicated that the introduced yeast-like fungus A. pullulans established in 2011 during fruit development and endured in the strawberry's phyllosphere for at least four weeks. As A. pullulans is a wide-spread colonizer of the phyllosphere and well adapted to this habitat [28], this result was anticipated. Likewise, the limited establishment of A. pullulans in 2012 is puzzling.

As the co-inoculated BCA, namely B. bassiana, did not establish epiphytically in the phyllosphere in both 2011 and 2012 (Figure 2), which is in accordance to the results from a previous study [22], we conclude that the co-application of the entomopathogenic fungus $B$. bassiana did not impair the establishment of $A$. pullulans. Indeed, the relative abundance of $A$. pullulans sometimes was significantly higher in multiple strain treatments than in single treatments with $A$. pullulans as determined by 454 pyrosequencing (Figure 7). In future experiments, it needs to be validated if co-application of B. bassiana might promote the establishment of $A$. pullulans, for instance indirectly by the potential endophytic growth of the co-applied fungus $B$. bassiana [37,38].

As for the resident microbial communities, the differences in the establishment of the model organism A. pullulans might be explained by (i) environmental factors and (ii) plant physiological factors [7,9], as well as (iii) the fitness of the introduced organism. In addition, introduced biological control agents have to compete with the resident microorganisms for nutrients and space to successfully immigrate and colonize the phyllosphere [10,11]. This paper illustrates the dynamics of both microbial residents and transients in the strawberry phyllosphere during the course of the crop. It might therefore be tempting to explain the present results by changing conditions during leaf maturation and shifting microbial functions of the residents. In this context, the role of Hymenobacter spp. and of representatives of the fungal order Helotiales would be interesting to further examine. Does Hymenobacter support the establishment of $A$. pullulans in the phyllosphere? Do representatives of Helotiales counteract the BCA's adhesion to the strawberry phyllosphere? 
Figure 7. Relative abundance of ITS rRNA sequences assigned to the genus Aureobasidium in strawberry leaf samples at $\mathrm{BBCH} 73$ (after four BCA applications) and $\mathrm{BBCH} 93$ (four weeks after the last BCA application) as influenced by treatments with Aureobasidium pullulans as a single treatment (AP) or co-inoculated with Beauveria bassiana (AP/BB) in 2011 and 2012. The control (W) was treated with water from a lotic reservoir. Error bars represent SEM values. Columns marked with the same letters are not significantly different within each phenological stage $(\alpha=0.05)$.
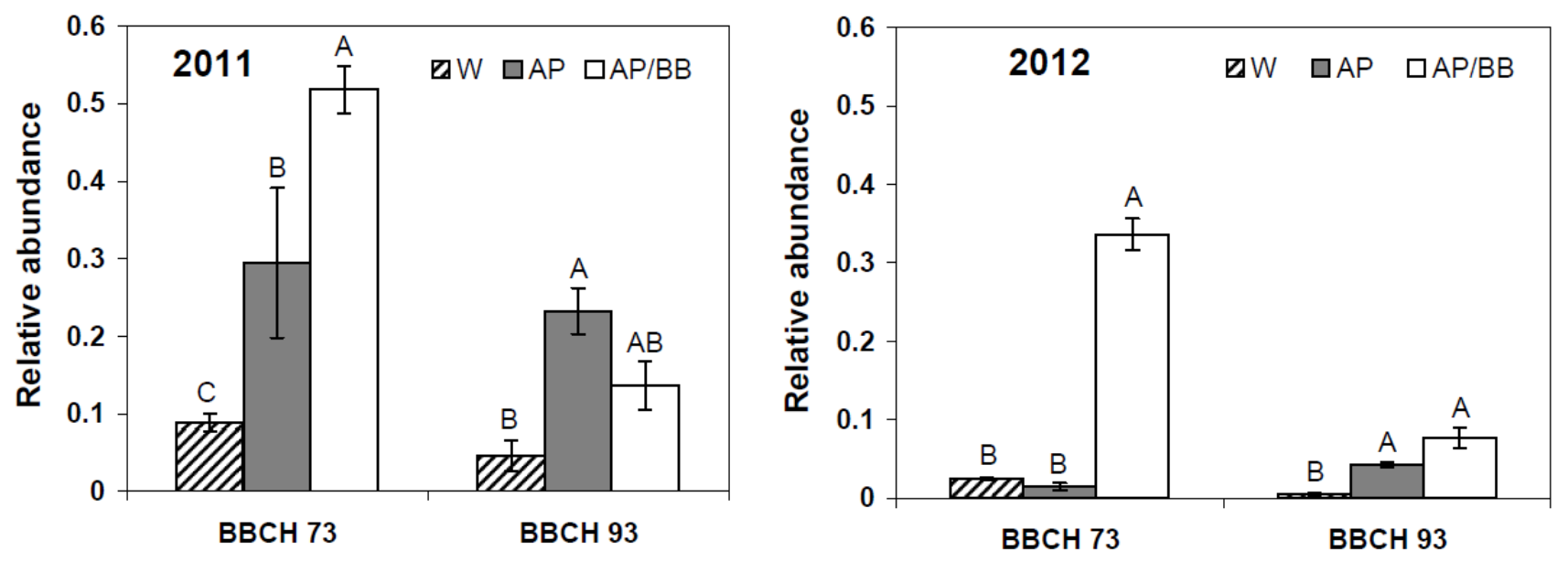

The results of the present study may also be seen in the light of the postulates of Cook et al. [17] on competitive displacement of non-target residents. As no plant pathogen was involved into the present study, the potential of such displacement caused by A. pullulans may be followed by the different treatments. Due to impaired establishment of the model organism during the second year of experiments (2012), the second year data set is not appropriate to conclude on the organism's short- or long-term impact and potential competitive displacement of non-target organisms in the strawberry phyllosphere. In the first experimental year (2011), plate counts indicated an increase in numbers of total culturable fungi in BCA-treated leaf samples shortly after repeated BCA applications, i.e., at $\mathrm{BBCH} 73$ (Figure 2) as compared to the untreated control. However, as the increased fungal counts probably resulted from the introduction of the yeast-like fungus, we suggest that foliar treatments with the model BCA as single strain treatment or co-inoculated with B. bassiana have neither a clear short-term nor a long-term impact on the culturable bacterial and fungal phyllosphere communities. These results are in line with findings from Russel et al. [24] and Sylla et al. [22].

Analysis using culture-independent tools verified the successful introduction of the model organism as the relative abundance of the order Dothideales and the genus Aureobasidium spp. was much more pronounced in BCA-treated leaf samples than in control samples after four applications of the BCAs (BBCH 73) in 2011 (Table 2 and Figure 2). Despite of comparable abundances in the remaining orders and genera in BCA-treated samples compared with the control plots, overall composition of fungal communities in the control samples differed from fungal communities in leaf samples previously treated with A. pullulans (ANOSIM: $p=0.027 ; \alpha=0.05 ; R=0.574$ ) and with $A$. pullulans co-inoculated with $B$. bassiana (ANOSIM: $p=0.030 ; \alpha=0.05 ; R=1$ ), displaying a significant decrease in fungal diversity shortly after the treatments with the respective BCAs (Figure 4A). Fungal richness was, however, not affected (Figure 4C). From the present data we conclude, that short-term 
displacement of the resident microbiome may occur as a consequence to introduction of $A$. pullulans at condition that the BCA establishes successfully. This needs to be verified in future studies, as this finding has not previously been reported for A. pullulans.

Four weeks after the adjourned BCA applications (BBCH 93) in 2011, the abundance of Dothideales was still increased in BCA-treated leaf samples (Table 2). Among all leaf samples, Cryptococcus and Aureobasidium made up the major fungal genera at this stage, whereas the abundance of Aureobasidium was highest in A. pullulans-treated leaf samples (Figure 3). At this stage, the fungal community composition was still significantly different in leaf samples previously treated with A. pullulans as single strain treatment as compared to control samples (ANOSIM: $p=0.029$; $\alpha=0.05 ; R=0.510$ ). This finding indicates that also long-term impacts of introduced A. pullulans strains on the phyllosphere microbiome may occur if the BCA establishes throughout the progression of the crop.

The yeast-like fungus $A$. pullulans is regarded as a promising candidate for biological control of nectrotrophic fungal pathogens (e.g., B. cinerea), which require high amounts of exogenous nutrients for germination and mycelial growth [32], as it strongly competes for nutrients [15]. We therefore suggest that the displacement of resident fungi might be explained by the good competitiveness of the established BCA in this habitat. It was also reported that other modes of action than competition for nutrients (e.g., induced resistance, enzyme production) might be generally involved in the biological control of plant pathogens [16,39] and, consequently, could be also involved in the interactions with the resident microorganisms. There is, however, still not enough knowledge if these mechanisms work in situ/in the field.

As 454 pyrosequencing data were insufficient for the leaf samples collected at $\mathrm{BBCH} 73$, i.e., after four BCA treatments, no conclusions on short-term impacts of introduced BCAs on the non-culturable bacterial communities could be made in 2011. Four weeks after the last BCA application (BBCH 93) in 2011, the composition (ANOSIM: $p=0.797 ; \alpha=0.05 ; R=-0.111$ ) as well as diversity and richness of bacterial communities was not significantly affected by previous treatments with A. pullulans as single strain treatment or co-inoculated with B. bassiana as compared to control leaf samples (Figure 6A,C). Interestingly, no long-term effects of the introduced BCAs on the bacterial communities in the phyllosphere were detected by means of 454 pyrosequencing in this study. This finding indicates that bacterial communities might be more stable than fungal communities in the phyllosphere of strawberries under field conditions, although one needs to keep in mind that the entire bacterial microbiota was probably not reflected in 2011. Likewise, Kim et al. [23] and Sylla et al. [22] did not observe any significant changes in bacterial communities by culture-independent techniques as a consequence of BCA applications under field conditions. In contrast, treatments with Bacillus thuringiensis significantly altered the composition of bacteria in the phyllosphere of greenhouse-grown pepper [21].

The results of the present study suggest that the co-application of $B$. bassiana does not clearly affect the interactions between $A$. pullulans and the resident strawberry leaf microbiota. 


\subsection{Interactions between Environmental Conditions and Microbial Dynamics in the Phyllosphere}

The phyllosphere is a highly open and dynamic environment, where microorganisms are strongly affected by environmental conditions such as rainfall, temperature and radiation $[7,18]$.

In the present study, the mean air temperatures did not considerably differ during the whole season in 2011 and 2012 (Table 6). In 2011, however, mean temperatures were higher three days prior to the third sampling than in 2012. Accumulated precipitation, however, was considerably higher during the entire growing season as well as the days prior to each leaf sampling in 2012 than in 2011.

Table 6. Temperatures and precipitation during the experiments in 2011 and 2012.

\begin{tabular}{cccccc}
\hline & \multicolumn{2}{c}{ Mean temperature [ $\left.{ }^{\circ} \mathbf{C}\right]$} & & \multicolumn{2}{c}{ Accumulated precipitation [mm] } \\
\cline { 2 - 3 } \cline { 5 - 6 } & $\mathbf{2 0 1 1}$ & $\mathbf{2 0 1 2}$ & & $\mathbf{2 0 1 1}$ & $\mathbf{2 0 1 2}$ \\
\hline During the whole season & $17.0^{\mathrm{a}}$ & $16.0^{\mathrm{a}}$ & & $103^{\mathrm{b}}$ & $153^{\mathrm{b}}$ \\
7 d prior to 1st sampling (BBCH 60) & $\mathrm{NA}^{\mathrm{c}}$ & 8.3 & & 3.0 & 7.3 \\
3 d prior to 1st sampling (BBCH 60) & $\mathrm{NA}^{\mathrm{c}}$ & 5.6 & & 0.0 & 2.3 \\
1 d prior to 1st sampling (BBCH 60) & $\mathrm{NA}^{\mathrm{c}}$ & 5.7 & & 0.0 & 2.0 \\
7 d prior to 2nd sampling (BBCH 73) & 14.4 & 14.9 & & 8.2 & 12.6 \\
3 d prior to 2nd sampling (BBCH 73) & 11.4 & 11.2 & & 0.0 & 0.6 \\
1 d prior to 2nd sampling (BBCH 73) & 9.8 & 10.3 & & 0.0 & 0.6 \\
7 d prior to 3rd sampling (BBCH 93) & 19.6 & 21.2 & & 7.4 & 14.1 \\
3 d prior to 3rd sampling (BBCH 93) & 24.3 & 18.8 & & 0.0 & 5.1 \\
1 d prior to 3rd sampling (BBCH 93) & 26.0 & 21.5 & & 0.0 & 0.0 \\
\hline
\end{tabular}

${ }^{\mathrm{a}}$ Temperature measurements from 12 April to the 3rd sampling date in both 2011 and 2012 (29 June 2011 and 4 July 2012) because in 2011 measurements started at 12 April; ${ }^{b}$ Data from 1 April to the 3rd sampling date (29 June 2011 and 4 July 2012) in both seasons; ${ }^{\mathrm{c}}$ No data available due to technical reasons.

High amounts of precipitation correlated negatively to the establishment of $A$. pullulans. In a short- and long-term perspective, the variations in Aureobasidium counts were explained to $27.4 \%$ by the total amount of precipitation and to $37.7 \%$ by the amount of precipitation seven days before leaf sampling $(p<0.001)$. In addition, the phyllosphere microbiomes differed between the two years as assessed by culture-dependent (Figures 1 and 2) and culture-independent methods (Tables 2-5), either with or without phyllosphere applied BCAs. This was most apparent for the genus Hymenobacter, which almost vanished during flowering in 2012, i.e., prior to BCA applications, as compared to the same phenological stage in the preceding year. When $A$. pullulans was introduced, either as single strain treatment or co-inoculated with B. bassiana, the abundance of the genus Aureobasidium was most conservative in 2011 as compared to the poor abundance of A. pullulans in 2012. Accordingly, the displacement effects on resident fungi in the year 2011 as determined by 454 pyrosequencing can be explained by the established $A$. pullulans strains. The observed differences in fungal communities between control leaf samples and leaf samples treated with $A$. pullulans co-inoculated with $B$. bassiana (ANOSIM: $p=0.029 ; \alpha=0.05 ; R=0.963$ ) at $\mathrm{BBCH} 73$, i.e., after four applications of the BCAs, however, cannot be explained by the introduced model BCA.

The interactions between phenological stages, weather conditions (precipitation and temperature) prior to sampling as well as microbial diversity and richness were also displayed by principal component analysis, where temperature and precipitation showed to have a strong influence on 
principal component (PC) 1 and PC 2, respectively (Figure 8). The samples for fungal ITS rRNA sequences were grouped according to the phenological growth stages in both 2011 and 2012 (Figure 8A), whereas the variation of the data could mainly be explained by temperature in 2011 and by both temperature and precipitation in 2012. The samples for 16S rRNA sequences were clearly separated at BBCH 93 between 2011 and 2012 (Figure 8B).

Figure 8. Principal component analysis (PCA) of interactivities in the phyllosphere microbiome of organically grown strawberries on the basis of Shannon diversity indices, Chaol estimators, temperature and precipitation data for (A) fungal ITS rRNA sequences and (B) bacterial 16S rRNA sequences at different phenological stages $(\bigcirc=\mathrm{BBCH} 60$, $\square=\mathrm{BBCH} 73, \diamond=\mathrm{BBCH}$ 93). Filled and open symbols represent data generated in 2011 and 2012, respectively. For 2011, data considering fungal ITS sequences and bacterial 16S rRNA sequences are excluded at $\mathrm{BBCH} 60$ due to missing temperature data. Data for bacterial 16S rRNA sequences are excluded at $\mathrm{BBCH} 73$ due to insufficient bioinformatic data.
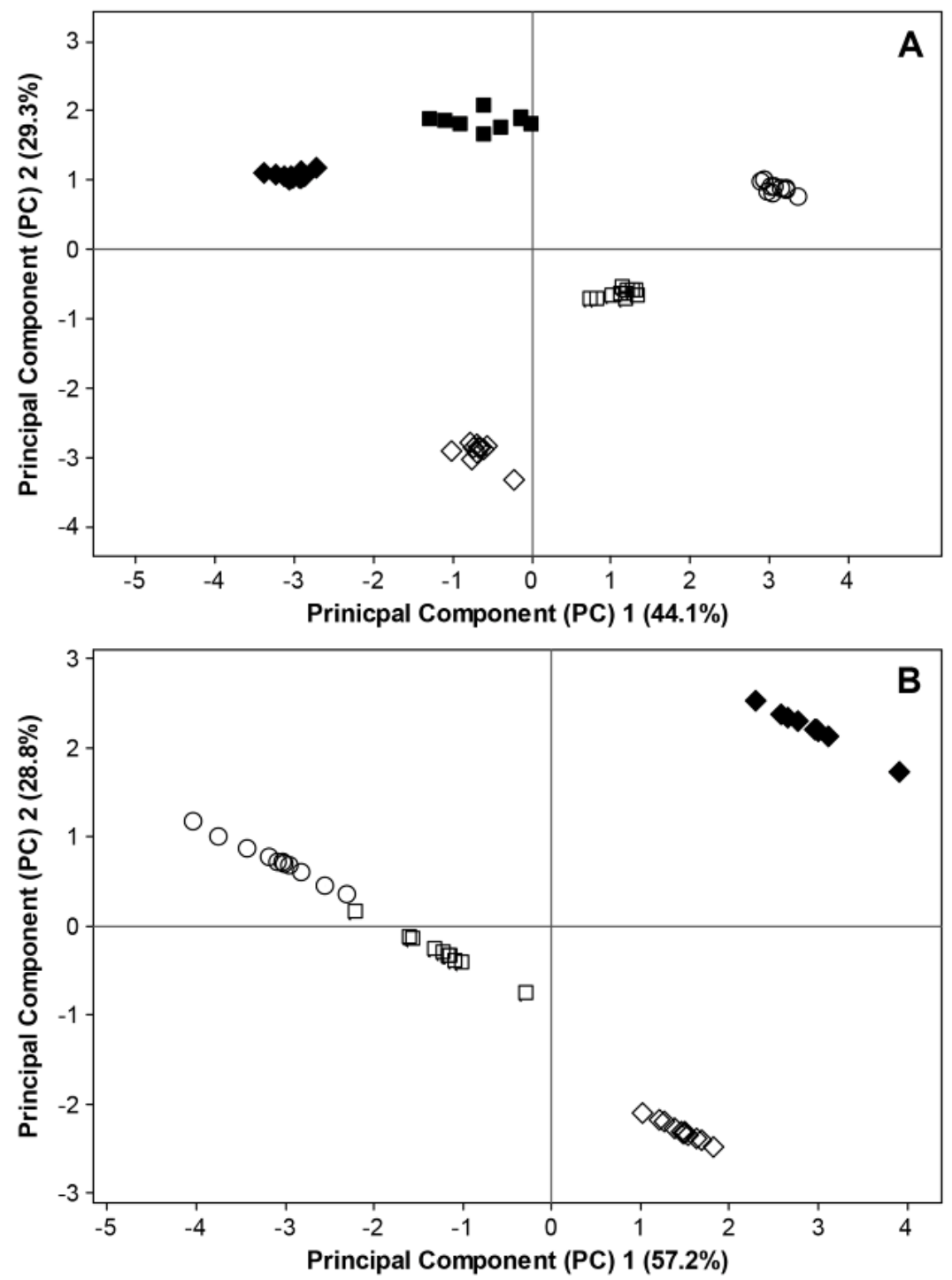
The strong dependence of community structure on environmental factors has repeatedly been shown $[40,41]$. As no data on boundary layer, nor on plant physiological parameters, were recorded in the present study, it is difficult to determine decisive factors or mechanisms. It is tempting to assume that the model organism was washed off. However, precipitation also affects light intensity and spectrum. At the present stage, it therefore would be unwise to ascribe the observed variations exclusively to mechanical interactions. We, therefore, recommend for future studies, to expand data collection to measurements of boundary layer conditions, further on-line monitoring of environmental conditions, including light intensities, light spectrum, UV-light, wind, and plant physiological responses.

An overhasty conclusion would be to reduce the discussion on the poor establishment of the model organism A. pullulans in the second experimental year to detrimental environmental conditions. It is worth to note, that the present experiment was conducted at the same site during two subsequent years and that the plants, from which the leaf samples were collected in the second year, were exposed for a second year to BCA treatments. Samples from flowering plants in year two (2012) displayed differences in their microbial community composition and structure between the three groups of planned treatments. No leaf samples were taken at a very early stage of the season which could have shed light on the intriguing question if these differences might be perennial long-term effects due to introduction of $A$. pullulans as a single or multiple strain treatment. This is an interesting question for the use of BCAs in perennial crops and should be further investigated.

The present study reveals new insights on the establishment of $A$. pullulans as a single or multiple strain treatment in a strawberry crop and to the inoculants' impact on the resident microbiome. Artificial inoculation of a plant pathogen and plant disease attack would by nature affect the resident microbial community composition and structure. Nevertheless, it points to two important new aspects that need to be included in future studies of short- and long-term effects of BCAs and to explain findings of impaired establishment of BCA, namely continuous monitoring of (i) meteorological and abiotic boundary layer parameters, as well as (ii) plant physiological parameters of importance for leaf colonization. For future studies, we furthermore recommend to consider more than three samplings to more effectively study the resident microbiota of the phyllosphere and the interactions with the introduced BCAs.

\section{Experimental Section}

\subsection{Experimental Design}

Experiments were conducted at Geisenheim University (Hesse, Geisenheim, Germany). Strawberry plants (Fragaria $\times$ ananassa Duch. cv. Elsanta) were planted in mid August 2010 and used for experiments in two fruiting seasons (2011 and 2012). After the first growing season in 2011, the crop was mulched. The plants were drip-irrigated on demand in both growing seasons and mulched with straw at the end of the flowering period in both 2011 and 2012. No other applications of plant protection products except the treatments described below were conducted to control pests and diseases.

From flowering through harvest, strawberry plants were treated weekly with single applications of the BCA preparation BoniProtect ${ }^{\circledR}$ forte $\left(7.5 \times 10^{9}\right.$ blastospores $\mathrm{g}^{-1}$ of the biological control agent 
Aureobasidium pullulans DSM 14940 and DSM 14941, bio-ferm $\mathrm{GmbH}$ ) and combined applications of the two BCA preparations BoniProtect ${ }^{\circledR}$ forte and Naturalis ${ }^{\circledR}\left(2.3 \times 10^{7}\right.$ conidia $\mathrm{mL}^{-1}$ of the entomo-pathogenic fungus Beauveria bassiana ATCC 74040, Intrachem Bio Deutschland GmbH \& Co.KG, Bad Camberg, Germany). The entomopathogenic fungus was included in these experiments because it showed high biocontrol potential against $B$. cinerea in preliminary tests on detached strawberry flowers (unpublished data). Depending on the plant development, the BCA preparations were applied eight times in 2011 and seven times in 2012, according to the manufacturer's instructions. Control plots were sprayed with surface water from a lotic reservoir (Rhine). Each treatment was applied to four plots consisting of each 80 plants, which were arranged in a randomized design in both 2011 and 2012. Air temperature $\left({ }^{\circ} \mathrm{C}\right)$ was measured at plant height $(\mathrm{ca} .30 \mathrm{~cm})$ at $10 \mathrm{~min}$ intervals by two data loggers (Macrologger 7000B, Unidata, O'Connor, Australia), which were distributed in the field. Information on the amount of precipitation $(\mathrm{mm})$ at Geisenheim was obtained from a meteorological weather station located in Geisenheim [42]. The mean temperature and accumulated precipitation was calculated for the whole season, 7 days prior to each sampling, 3 days prior to each sampling, and 1 day prior to each sampling, respectively (Table 6).

\subsection{Sample Collection and Microbe Extraction}

Leaf samples were collected at three phenological stages of strawberries as determined by the $\mathrm{BBCH}$-identification key of Meier et al. [43]; (i) $\mathrm{BBCH} 60$ (first flowers open, i.e., before the first BCA application); (ii) $\mathrm{BBCH} 73$ (seeds visible on receptacle tissue/fruit-setting, i.e., plants were already treated four times with the respective BCAs); and (iii) $\mathrm{BBCH} 93$ (old leaves dying, i.e., plants have not been treated with BCAs for four weeks). Leave samples collected at BBCH 93 were, thus, an indicator for long-term effects of BCAs. At all samplings, one leaf per plant was carefully sampled from a total of 30 plants in each plot to determine the microbial communities in the strawberry phyllosphere. This corresponded to four replicates per sample with each 30 pooled leaves. Leaf samples were stored on ice and transported to the laboratory where they were weighed and transferred into sterile glass flasks $(250 \mathrm{~mL})$. Each flask was filled with aliquots of $230 \mathrm{~mL}$ of PBS buffer (8 g liter ${ }^{-1} \mathrm{NaCl}, 0.2 \mathrm{~g} \mathrm{liter}^{-1} \mathrm{KCl} ; 1.44 \mathrm{~g} \mathrm{liter}^{-1} \mathrm{Na}_{2} \mathrm{HPO}_{4} ; 0.24 \mathrm{~g} \mathrm{liter}^{-1} \mathrm{KH}_{2} \mathrm{PO}_{4} ; \mathrm{pH}=7.4$ ) and shaken on an overhead shaker (30 min at ca. $50 \mathrm{rpm}$ ) followed by sonication ( $7 \mathrm{~min}$ at $35 \mathrm{kHz}$, Bandelin Sonorex Super RK510H) for washing-off the microorganisms from the leaves. The resulting wash solutions were transferred into $250 \mathrm{~mL}$ centrifuge flasks and used for plate counts and DNA isolation.

\subsection{Plate Counts}

From each wash solution, aliquots of $50 \mu \mathrm{L}$ (undiluted) were spiral-plated (Whitley Automatic Spiral Plater, Don Whitley Scientific, New Yorkshire, England) on various nutrient media. For the determination of the colony-forming units (CFU) of total bacteria and total fungi, wash solutions were plated on R2A (18.2 $\mathrm{g}_{\text {liter }}{ }^{-1}$ Difco $^{\mathrm{TM}} \mathrm{R} 2 \mathrm{~A}$ agar, BD, Le Pont de Claix, France; $0.1 \mathrm{~g} \mathrm{liter}^{-1}$ cycloheximide) and diluted PDA (10.0 $\mathrm{g} \mathrm{liter}^{-1}$ potato dextrose agar, Merck, Darmstadt, Germany; $12.0 \mathrm{~g}_{\text {liter }^{-1}}$ Bacto $^{\mathrm{TM}}$ agar, BD, Le Pont de Claix, France; $0.1 \mathrm{~g}$ liter $^{-1}$ streptomycin sulphate; $0.01 \mathrm{~g} \mathrm{liter}^{-1}$ tetracycline hydrochloride). The CFU of endospore-forming bacteria was determined by 
heating aliquots of $5 \mathrm{ml}$ from each wash solution $\left(80^{\circ} \mathrm{C}\right.$ for $\left.15 \mathrm{~min}\right)$ followed by plating on 0.1 TSA (4.0 $\mathrm{g} \mathrm{liter}^{-1}$ Difco $^{\mathrm{TM}}$ tryptic soy agar, BD, Le Pont de Claix, France; $13.5 \mathrm{~g} \mathrm{liter}^{-1}$ Bacto $^{\mathrm{TM}}$ agar, BD, Le Pont de Claix, France; $0.1 \mathrm{~g} \mathrm{liter}^{-1}$ cycloheximide). The amount of Beauveria spp. was determined by plating each wash solution on Beauveria selective medium (18.0 $\mathrm{g} \mathrm{liter}^{-1}$ Bacto $^{\mathrm{TM}}$ agar, BD, Le Pont de Claix, France; $20.0 \mathrm{~g} \mathrm{liter}^{-1}$ glucose; $10.0 \mathrm{~g} \mathrm{liter}^{-1}$ soya peptone; $0.05 \mathrm{~g} \mathrm{liter}^{-1}$ cycloheximide; $0.1 \mathrm{~g} \mathrm{liter}^{-1}$ dodine; $0.1 \mathrm{~g}$ liter $^{-1}$ streptomycin sulphate; $0.05 \mathrm{~g} \mathrm{liter}^{-1}$ tetracycline hydrochloride). For determining the CFU of Aureobasidium spp. each wash solution was plated on SA $\left(65.0 \mathrm{~g} \mathrm{liter}^{-1}\right.$ $\mathrm{BBL}^{\mathrm{TM}}$ Sabouraud dextrose agar, BD, Le Pont de Claix, France; $0.3 \mathrm{~g} \mathrm{liter}^{-1}$ streptomycin sulphate) and colonies of Aureobasidium spp. were morphologically identified. The results of the plate counts were expressed as CFU per g leaf dry weight (DW).

\subsection{DNA Isolation and 454 Pyrosequencing}

The remaining wash solutions were processed by centrifugation $\left(14,000 \times g\right.$ at $4{ }^{\circ} \mathrm{C}$ for $\left.20 \mathrm{~min}\right)$ and discarding of the supernatant. Genomic DNA was extracted from the resulting pellet using the Power Soil DNA Isolation Kit (Sued-Laborbedarf GmbH, Gauting, Germany) according to the manufacturer's instructions. The amplification of fragments of fungal ITS rRNA gene and bacterial 16S rRNA gene was performed using the primer pairs ITS1 and ITS2 [44], and 27F and 337R [45], respectively.

For 454 pyrosequencing, the GS FLX Titanium Primers A (CGTATCGCCTCCCTCGCGCCA) and B (CTATGCGCCTTGCCAGCCCGC) as well as a key sequence (TCAG) were added to the universal primers. For barcoding, a sample specific multiplex identifier sequence (MID) was added between the Primer A and B and the universal primers. Primers with different MID-tags were individually used in amplification reactions of each sample replicate from a total of 72 samples (3 treatments with 4 replicates, 3 sampling dates both in 2011 and 2012).

Aliquots of $0.2 \mu \mathrm{L}$ Dream Taq ${ }^{\mathrm{TM}}$ DNA Polymerase ( $5 \mathrm{U}_{\mu} \mathrm{L}^{-1}$; Fermentas, St. Leon-Rot, Germany), $2 \mu \mathrm{L}$ Dream Taq ${ }^{\mathrm{TM}}$ Buffer (10×; Fermentas, St. Leon-Rot, Germany), $0.4 \mu \mathrm{L}$ dNTPs (10 mM; Fermentas, St. Leon-Rot, Germany), $1 \mu \mathrm{L}$ primers (10 pmol) and $20 \mathrm{ng}$ of genomic DNA were added to the PCR reaction mixtures in a final volume of $20 \mu \mathrm{L}$. For amplifying ITS rRNA and 16S rRNA genes, the PCR parameters were set according to [44,46], respectively. Before purifying the PCR products using the HiYield PCR Clean-up/Gel Extraction Kit (Sued-Laborbedarf GmbH, Gauting, Germany), aliquots of $4 \mu \mathrm{L}$ of each PCR product were loaded on a $1 \%$ agarose gel and tested for successful amplification and correct size ( $\sim 400 \mathrm{bp}$ and $\sim 450 \mathrm{bp}$ for fungal ITS rRNA and bacterial 16S rRNA PCR products, respectively). Fungal and bacterial PCR products were pooled at equal molar concentrations, respectively. Both amplicon sample pools were sent to a commercial laboratory (LGC Genomics GmbH, Berlin, Germany) for 454 pyrosequencing using Roche/454 Genome Sequencer FLX + Titanium.

\subsection{OTU Picking and Taxomomic Assignment}

Analyses of 454 pyrosequencing data were performed by the bioinformatic services of LGC Genomics (Berlin, Germany) using the QIIME Virtual Box version 1.6.0 [47]. The 454 pyrosequencing reads were demultiplexed and clipped followed by denoizing of the reads (removing 
of erroneous reads) with AmpliconNoise [48]. Parameters used for quality filtering during the denoising step were set as follows: maximum read length: $450 \mathrm{bp}$; seqnoise_resolution: 30.0 .

Operational taxonomic units (OTUs) were picked by clustering sequences at a similarity of $>97 \%$. From each OTU, the most abundant sequence was selected as the representative sequence and chimeras were excluded from alignment using ChimeraSlayer. Representative sequences of OTUs from the 16S rRNA sample pool were aligned with reference sequences from the Greengenes database (version 12_10), whereas those from the ITS rRNA sample pool were aligned with reference sequences from the UNITE database (version 12_11; alpha release) using the RPD classifier [49] at $80 \%$ minimum confidence. Samples with less than 50 reads were excluded from further analysis.

\subsection{Statistical Analysis of Data from Plate Counts and 454 Pyrosequencing}

Plate count data were analyzed with the STATISTICA software package, version 7.1 [50]. Plate counts were log-transformed $\left(x^{\prime}=x+1\right)$ and tested for normal distribution (Shapiro-Wilk-Test, $\alpha=0.05)$. A Mann-Whitney-U-Test was performed for comparing plate counts of the resident microbial groups on flowering plants (BBCH 60) between 2011 and $2012(\alpha=0.05)$. A one-way ANOVA or a non-parametric Kruskal-Wallis-ANOVA were performed for normally and not normally distributed data, respectively, followed by Tukey-Test or multiple comparisons using Kruskal-Wallis-Test to compare plate counts of differentially treated samples within each microbial group at $\mathrm{BBCH} 73$ and $\mathrm{BBCH} 93$, respectively (each at $\alpha=0.05$ ).

Using the paleontological statistics software package (PAST), version 2.17b [51], the Shannon indices and richness estimators (Chao1) were calculated for the abundances of OTUs (absolute counts). For comparing the composition of fungal and bacterial communities in differentially treated leaf samples at each sampling date, analyses of similarity (ANOSIM, with 9999 permutations) were calculated in PAST based on Bray-Curtis similarities of both fungal and bacterial OTUs (\% reads), respectively. In addition to $p$ values, ANOSIM creates $R$ values which usually range from $0-1$ ( $R$ values close to 0 indicate high similarity and those close to 1 indicate low similarity between the microbial communities of different leaf samples). The standard statistical analyses of 454 pyrosequencing data were performed using STATISTICA. The data for Shannon and Chao 1 indices as well as for the relative abundance of Aureobasidium reads were normally distributed (Shapiro-Wilk-Test, $\alpha=0.05$ ). A one-way ANOVA followed by Tukey-Test $(\alpha=0.05)$ was performed to detect differences in the Shannon indices, Chaol estimators and the abundances of Aureobasidium reads between differentially treated leaf samples at each sampling date. Calculation of correlations, regressions as well as principal component analysis (PCA) was performed with Minitab statistical software, version 16.1.0.0 [52]. The latter one included Shannon diversity indices, Chao1 estimators as well as weather data.

\section{Conclusions}

Varying consistencies of BCAs have been demonstrated in various studies and impose a substantial concern for the users of biological control agents both in integrated and organic production $[3,5,53,54]$. The present study demonstrates that the road to success is very complex. Establishment of introduced organisms in a novel habitat is dependent on multiple factors (e.g., environmental conditions, 
microbial community structure). Furthermore, the resident microbiome itself considerably changes depending on environmental and plant physiological factors. Therefore, it is difficult to appoint exclusive interactions between the introduced model BCA and the dynamic resident microbial communities in the phyllosphere. At the present stage of this study we, therefore, conclude that the observed short-term and long-term effects on fungal communities caused by the introduced A. pullulans strains can be considered as negligible under practical conditions.

\section{Acknowledgments}

Many thanks to bio-ferm $\mathrm{GmbH}$ and Intrachem Bio Deutschland $\mathrm{GmbH} \& \mathrm{Co} . \mathrm{KG}$ for providing the BCA preparations. We also thank Sonja Stutzke, Martha Schott, the employees at the Department of Pomology (Geisenheim University) as well as all involved student assistants for technical assistance. The project is supported by funds of the Federal Ministry of Food, Agriculture and Consumer Protection (BMELV) based on a decision of the Parliament of the Federal Republic of Germany via the Federal Office for Agriculture and Food (BLE) under the innovation support program, which is gratefully acknowledged. The present study was performed within the framework of the postgraduate school $\mu$ HORT, funded by the Swedish research council Formas, which is also acknowledged.

\section{Conflicts of Interest}

The authors declare no conflict of interest.

\section{References}

1. Elad, Y.; Kirshner, B.; Yehuda, N.; Sztejnberg, A. Management of powdery mildew and gray mold of cucumber by Trichoderma harzianum T39 and Ampelomyces quisqualis AQ10. BioControl 1998, 43, 241-251.

2. Kovach, J.; Petzoldt, R.; Harman, G.E. Use of honey bees and bumble bees to disseminate Trichoderma harzianum 1295-22 to strawberries for Botrytis control. Biol. Control 2000, 18, 235-242.

3. Elad, Y.; Stewart, A. Microbial Control of Botrytis spp. In Botrytis: Biology, Pathology and Control; Elad, Y., Williamson, B., Tudzynski, P., Delen, N., Eds.; Kluwer Academic Publishers: Dordrecht, The Netherlands, 2004; pp. 223-241.

4. Freeman, S.; Minz, D.; Kolesnik, I.; Barbul, O.; Zveibil, A.; Maymon, M.; Nitzani, Y.; Kirshner, B.; Rav-David, D.; Bilu, A.; et al. Trichoderma biocontrol of Colletotrichum acutatum and Botrytis cinerea and survival in strawberry. Eur. J. Plant Pathol. 2004, 110, 361-370.

5. Prokkola, S.; Kivijärvi, P. Effect of biological sprays on the incidence of grey mould, fruit yield and fruit quality in organic strawberry production. Agric. Food Sci. 2007, 16, 25-33.

6. Pertot, I.; Zasso, R.; Amsalem, L.; Baldessari, M.; Gino Angeli, G.; Elad, Y. Integrating biocontrol agents in strawberry powdery mildew control strategies in high tunnel growing systems. Crop Prot. 2008, 27, 622-631.

7. Andrews, J.H. Biological control in the phyllosphere. Annu. Rev. Phytopathol. 1992, 30, 603-635. 
8. Lindow, S.E.; Brandl, M.T. Microbiology of the phyllosphere. Appl. Environ. Microbiol. 2003, 69, 1875-1883.

9. Whipps, J.M.; Hand, P.; Pink, D.; Bending, G.D. Phyllosphere microbiology with special reference to diversity and plant genotype. J. Appl. Microbiol. 2008, 105, 1744-1755.

10. Jacobsen, B.J. Biological Control of Plant Diseases by Phyllosphere Applied Biological Control Agents. In Microbial Ecology of Aerial Plant Surfaces; Bailey, M.J., Lilley, A.K., Timms-Willson, T.M., Spencer-Phillips, P.T.N., Eds.; CABI: Oxfordshire, UK, 2006; pp. 133-147.

11. Elad, Y.; Kirshner, B. Survival in the phylloplane of an introduced biocontrol agent (Trichoderma harzianum) and populations of the plant pathogen Botrytis cinerea as modified by abiotic conditions. Phytoparasitica 1993, 21, 303-313.

12. Monier, J.M.; Lindow, S.E. Aggregates of resident bacteria facilitate survival of immigrant bacteria on leaf surfaces. Microb. Ecol. 2005, 49, 343-352.

13. Longa, C.M.O.; Pertot, I.; Tosi, S. Ecophysiological requirements and survival of a Trichoderma atroviride isolate with biocontrol potential. J. Basic Microbiol. 2008, 48, 269-277.

14. Guetsky, R.; Shtienberg, D.; Dinoor, A.; Elad, Y. Establishment, survival and activity of the biocontrol agents Pichia guilermondii and Bacillus mycoides applied as a mixture on strawberry plants. Biocontrol Sci. Technol. 2002, 12, 705-714.

15. Lima, G.; Ippolito, A.; Nigro, F.; Salerno, M. Effectiveness of Aureobasidium pullulans and Candida oleophila against postharvest strawberry rots. Postharvest Biol. Technol. 1997, 10, 169-178.

16. Adikaram, N.; Joyce, D.; Terryc, L. Biocontrol activity and induced resistance as a possible mode of action for Aureobasidium pullulans against grey mould of strawberry fruit. Australas. Plant Pathol. 2002, 31, 223-229.

17. Cook, J.; Bruckart, W.L.; Coulson, J.R.; Goettel, M.S.; Humber, R.A.; Lumsden, R.D.; Maddox, J.V.; McManus, M.L.; Moore, L.; Meyer, S.F.; et al. Safety of microorganisms intended for pest and plant disease control: A framework for scientific evaluation. Biol. Control 1996, 7 , 333-351.

18. Kinkel, L.L. Microbial population dynamics on leaves. Annu. Rev. Phytopathol. 1997, 35, 327-347.

19. Hunter, P.J.; Hand, P.; Pink, D.; Whipps, J.M.; Bending, G.D. Both leaf properties and microbe-microbe interactions influence within-species variation in bacterial population diversity and structure in the lettuce (Lactuca species) phyllosphere. Appl. Environ. Microbiol. 2010, 76, 8117-8125.

20. De Jager, E.S.; Wehner, F.C.; Korsten, L. Microbial ecology of the mango phylloplane. Microb. Ecol. 2001, 42, 201-207.

21. Zhang, B.; Bai, Z.; Hoefel, D.; Tang, L.; Yang, Z.; Zhuang, G.; Yang, J.; Zhang, H. Assessing the impact of the biological control agent Bacillus thuringiensis on the indigenous microbial community within the pepper plant phyllosphere. FEMS Microbiol. Lett. 2008, 284, 102-108.

22. Sylla, J.; Alsanius, B.W.; Krüger, E.; Reineke, A.; Strohmeier, S.; Wohanka, W. Leaf microbiota of strawberries as affected by biological control agents. Phytopathology 2013, 103, 1001-1011.

23. Kim, Y.; Cho, M.; Jeong, J.; Lee, H.; Kim, S. Application of terminal restriction fragment length polymorphism (T-RFLP) analysis to monitor effect of biocontrol agents on rhizosphere microbial community of hot pepper (Capsicum annuum L.). J. Microbiol. 2010, 48, 566-572. 
24. Russell, D.; Chard, J.; McKinlay, R. Effect of Bacillus thuringiensis and a pyrethroid insecticide on the leaf microflora of Brassica oleracea. Lett. Appl. Microbiol. 1999, 28, 359-362.

25. Okon Levy, N.; Elad, Y.; Katan, J.; Baker, S.C.; Faull, J.L. Trichoderma and soil solarization induced microbial changes on plant surfaces. IOBC/WPRS Bull. 2006, 29, 21-26.

26. Deacon, J. Fungal Ecology: Saprotrophs. In Fungal biology, 4th ed.; Blackwell Publishing: Malden, MA, USA, 2006; pp. 213-236.

27. Andrews, J.H. Population Growth and the Landscape Ecology of Microbes on Leaf Surfaces. In Microbial Ecology of Aerial Plant Surfaces; Bailey, M.J., Lilley, A.K., Timms-Willson, T.M., Spencer-Phillips, P.T.N., Eds.; CABI: Oxfordshire, UK, 2006; pp. 239-250.

28. Chi, Z.; Wang, F.; Chi, Z.; Yue, L.; Liu, G.; Zhang, T. Bioproducts from Aureobasidium pullulans, a biotechnologically important yeast. Appl. Microbiol. Biotechnol. 2009, 82, 793-804.

29. Thompson, I.P.; Bailey, M.J.; Fenlon, J.S.; Fermor, T.R.; Lilley, A.K.; Lynch, J.M.; McCormack, P.J.; McQuilken, M.P.; Purdy, K.J.; Rainey, P.B.; et al. Quantitative and qualitative seasonal changes in the microbial community from the phyllosphere of sugar beet (Beta vulgaris). Plant Soil 1993, 150, 177-191.

30. Inácio, J.; Pereira, P.; Carvalho, M.; Fonseca, Á.; Amaral-Collaço, M.T.; Spencer-Martins, I. Estimation and diversity of phylloplane mycobiota on selected plants in a Mediterranean-type ecosystem in Portugal. Microb. Ecol. 2002, 44, 344-353.

31. Jensen, B.; Knudsen, I.M.B.; Andersen, B.; Nielsen, K.F.; Thrane, U.; Jensen, D.F.; Larsen, J. Characterization of microbial communities and fungal metabolites on field grown strawberries from organic and conventional production. Int. J. Food Microbiol. 2013, 160, 313-322.

32. Blakeman, J.P.; Fokkema, N.J. Potential for biological control of plant diseases on the phylloplane. Annu. Rev. Phytopathol. 1982, 20, 167-190.

33. Leveau, J.H.J.; Tech, J.J. Grapevine microbiomics: Bacterial diversity on grape leaves and berries revealed by high-throughput sequence analysis of 16S rRNA amplicons. Acta Hortic. 2011, 905, $31-42$.

34. Ottesen, A.R.; White, J.R.; Skaltsas, D.N.; Newell, M.J.; Walsh, C.S. Impact of organic and conventional management on the phyllosphere microbial ecology of an apple crop. J. Food Prot. 2009, 72, 2321-2325.

35. Redford, A.J.; Fierer, N. Bacterial succession on the leaf surface: A novel system for studying successional dynamics. Microb. Ecol. 2009, 58, 189-198.

36. Kitajima, K.; Mulkey, S.; Wright, S. Decline of photosynthetic capacity with leaf age in relation to leaf longevities for five tropical canopy tree species. Am. J. Bot. 1997, 84, 702-708.

37. Tefera, T.; Vidal, S. Effect of inoculation method and plant growth medium on endophytic colonization of sorghum by the entomopathogenic fungus Beauveria bassiana. BioControl 2009 , 54, 663-669.

38. Vega, F.E.; Posada, F.; Catherine Aime, M.; Pava-Ripoll, M.; Infante, F.; Rehner, S.A. Entomopathogenic fungal endophytes. Biol. Control 2008, 46, 72-82.

39. Castoria, R.; de Curtis, F.; Lima, G.; Caputo, L.; Pacifico, S.; de Cicco, V. Aureobasidium pullulans (LS-30) an antagonist of postharvest pathogens of fruits: Study on its modes of action. Postharvest Biol. Technol. 2001, 22, 7-17. 
40. Kadivar, H.; Stapleton, A.E. Ultraviolet radiation alters maize phyllosphere bacterial diversity. Microb. Ecol. 2003, 45, 353-361.

41. Finkel, O.M.; Burch, A.Y.; Lindow, S.E.; Post, A.F.; Belkin, S. Geographical location determines the population structure in phyllosphere microbial communities of a salt-excreting desert tree. Appl. Environ. Microbiol. 2011, 77, 7647-7655.

42. Wetteronline. Available online: www.wetteronline.de (accessed on 29 May 2013).

43. Meier, U.; Graf, H.; Hack, H.; Hess, M.; Kennel, W.; Klose, R.; Mappes, D.; Seipp, D.; Stauss, R.; Streif, J.; et al. Phänologische Entwicklungsstadien des Kernobstes (Malus domestica Borkh.und Pyrus communis L.), des Steinobstes (Prunus-Arten), der Johannisbeere (Ribes-Arten) und der Erdbeere (Fragaria x ananassa Duch.). Nachrichtenbl. Deut. Pflanzenschutzd. 1994, 46, 141-153.

44. Buée, M.; Reich, M.; Murat, C.; Morin, E.; Nilsson, R.H.; Uroz, S.; Martin, F. 454 pyrosequencing analyses of forest soils reveal an unexpectedly high fungal diversity. New Phytol. 2009, 184, 449-456.

45. Hamp, T.J.; Jones, W.J.; Fodor, A.A. Effects of experimental choices and analysis noise on surveys of the "rare biosphere". Appl. Environ. Microbiol. 2009, 75, 3263-3270.

46. Hirsch, J.; Strohmeier, S.; Pfannkuchen, M.; Reineke, A. Assessment of bacterial endosymbiont diversity in Otiorhynchus spp. (Coleoptera: Curculionidae) larvae using a multitag 454 pyrosequencing approach. BMC Microbiol. 2012, 12, S6.

47. Caporaso, J.G.; Kuczynski, J.; Stombaugh, J.; Bittinger, K.; Bushman, F.D.; Costello, E.K.; Fierer, N.; Peña, A.G.; Goodrich, J.K.; Gordon, J.I.; et al. QIIME allows analysis of high-throughput community sequencing data. Nat. Methods 2010, 7, 335-336.

48. Quince, C.; Lanzén, A.; Curtis, T.P.; Davenport, R.J.; Hall, N.; Head, I.M.; Read, L.F.; Sloan, W.T. Accurate determination of microbial diversity from 454 pyrosequencing data. Nat. Methods 2009, 6, 639-641.

49. Wang, Q.; Garrity, G.M.; Tiedje, J.M.; Cole, J.R. Naive Bayesian classifier for rapid assignment of rRNA sequences into the new bacterial taxonomy. Appl. Environ. Microbiol. 2007, 73, 5261-5267.

50. STATISTICA für Windows [Software-System für Datenanalyse], Version 7.1.; StatSoft, Inc.: Tulsa, OH, USA, 2005.

51. Hammer, Ø.; Harper, D.A.T.; Ryan, P.D. PAST: Paleontological statistics software package for education and data analysis. Palaeontol. Electron. 2001, 4, 1-9.

52. Minitab, I. Minitab 16 Statistical Software. Available online: www.minitab.com (acessed on 1 September 2012).

53. Alabouvette, C.; Olivain, C.; Steinberg, C. Biological control of plant diseases: The european situation. Eur. J. Plant Pathol. 2006, 114, 329-341.

54. Moser, R.; Pertot, I.; Elad, Y.; Raffaelli, R. Farmers' attitudes toward the use of biocontrol agents in IPM strawberry production in three countries. Biol. Control 2008, 47, 125-132.

(C) 2013 by the authors; licensee MDPI, Basel, Switzerland. This article is an open access article distributed under the terms and conditions of the Creative Commons Attribution license (http://creativecommons.org/licenses/by/3.0/). 